\begin{tabular}{|c|c|}
\hline 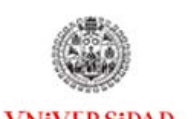 & $\begin{array}{l}\text { Teoría de la Educación } \\
\text { Educación y Cultura en la }\end{array}$ \\
\hline $\begin{array}{l}\text { VNiVERSIDAD } \\
\text { BSALAMANCA }\end{array}$ & Sociedad de la Información \\
\hline
\end{tabular}

\title{
BLENDED-LEARNING E INGENIERÍA: NIVEL DE USO, RENDIMIENTO ACADÉMICO Y VALORACIÓN DE LOS ALUMNOS
}

\author{
Ana Isabel VÁZQUEZ-MARTÍNEZ \\ Universidad de Sevilla \\ aisabel@us.es
}

\author{
Juan Manuel ALDUCIN-OCHOA \\ Universidad de Sevilla \\ alducin@us.es
}

\section{Resumen:}

Los cambios que está experimentando la universidad, así como las características de las enseñanzas técnicas, en cuanto a alta tasa de abandono y el bajo rendimiento académico, hacen que se tengan que buscar soluciones para dar respuesta a estos retos. En este artículo se presenta el empleo de la plataforma educativa WebCT en la asignatura Materiales de Construcción-I, en la titulación de Ingeniería de Edificación de la Universidad de Sevilla. Se pretende analizar cuál es el nivel de uso, el rendimiento académico y la valoración que los estudiantes hacen de su uso. De esta manera los objetivos planteados son conocer si el empleo de las herramientas foro y pruebas preparatorias ejerce influencia en el rendimiento de los estudiantes; si existe relación entre el rendimiento y la valoración que los estudiantes hacen de WebCT, y entre el empleo de las herramientas citadas y la valoración dada. Los resultados indican que existen diferencias significativas en el rendimiento académico por el grado de empleo de las pruebas y por el grado de empleo del foro, así como entre la percepción y satisfacción de los estudiantes según el grado de empleo de pruebas y foro. De la misma manera existe correlación entre el rendimiento académico y la valoración que hacen los estudiantes del empleo de WebCT. Por ello se podría decir que la enseñanza presencial apoyada en plataformas educativas puede promover cambios significativos en los resultados académicos, siendo al mismo tiempo un recurso bien valorado por los estudiantes.

Palabras clave: blended learning, Ingeniería, rendimiento académico, valoración. 


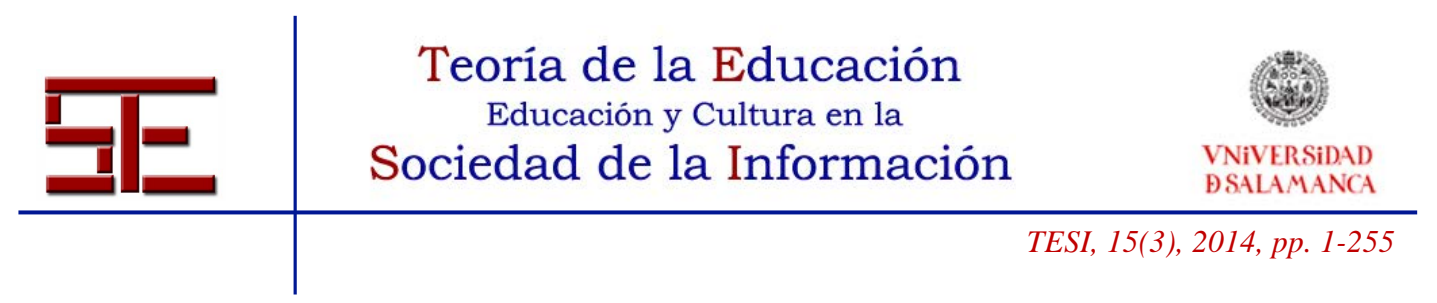

\section{BLENDED LEARNING AND ENGINEERING: USE LEVEL, ACADEMIC PERFORMANCE AND ASSESSMENT OF STUDENTS}

Abstract:

Changes that the University is experiencing, as well as the characteristics of technical teachings, in terms of high drop-out rates and low academic performance, do that they have to seek solutions to respond to these challenges. This article presents the use of the learning platform WebCT course Materials of Construccion-I, in the degree of Engineering of Building of the University of Seville. Intends to analyze what is the level of use, the academic performance and the valuation that students make their use. In this way the objectives are to know if the use of the tools forum and preparatory tests exerts influence on the performance of students; if there is a relationship between the performance and the valuation that students make WebCT, and between the use of these tools and the given valuation. The results indicate that there are significant differences in academic achievement by the degree of use of evidence and the degree of use of the Forum, as well as between perception and satisfaction of the Teas study according to the degree of use of evidence and forum. In the same way there is correlation between academic performance and the evaluation and satisfaction of students. Therefore one could say that classroom teaching supported by educational platforms can promote significant changes in academic results, being at the same time a resource well valued by students

Key words: blended learning, Engineering, academic performance, assessment. 


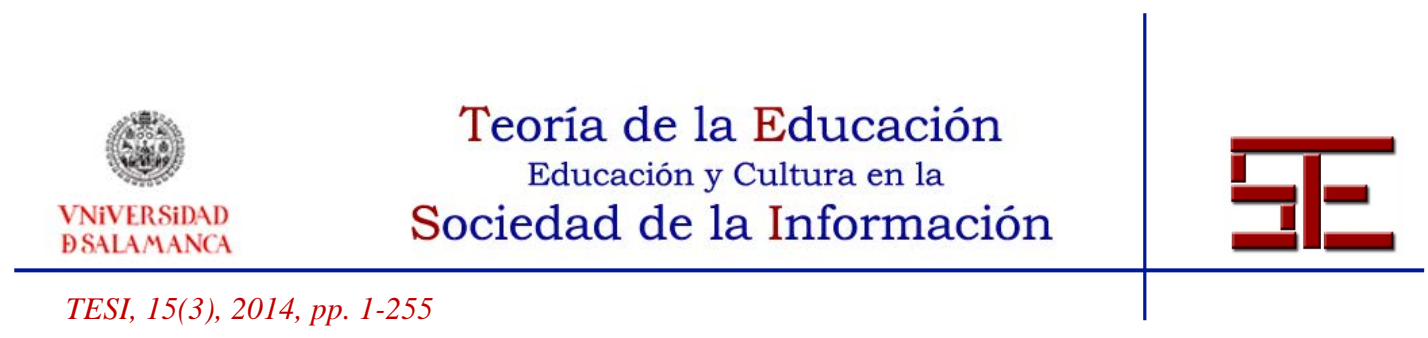

\section{INTRODUCCIÓN}

Las tendencias en la educación universitaria actual se sustenta en dos pilares básicos: la incorporación al Espacio Europeo de Educación Superior (EEES) y la implementación del European Credit Transfer System (ECTS).

El EEES ha generado grandes expectativas dado que la universidad no solo debe enseñar a saber, sino también enseñar a aprender, enseñar a hacer, enseñar a ser. Como bien señala García Peñalvo (2008, p. 30) al respecto "el alumno es el centro de su propio aprendizaje. En este modelo los estudiantes son activos y participativos, aprenden a su propio ritmo y usan sus propias estrategias; están más intrínseca que extrínsecamente motivados, y el aprendizaje es más individualizado que estandarizado". De igual manera, de Miguel (2004) nos recuerda que la implantación del EEES debe "promover el cambio metodológico de una enseñanza centrada sobre la actividad del profesor a otra orientada hacia el aprendizaje del alumno. No se trata de distribuir los contenidos a lo largo de un cronograma o distribución horaria, sino de tutorizar secuencialmente las experiencias de aprendizaje de los estudiantes guiando sus aprendizajes" (p. 58).

La adopción del ECTS promueve la valoración de manera global del trabajo desarrollado por los estudiantes, y el seguimiento de sus aprendizajes por parte de los docentes. El Real Decreto 1125/2003, por el que se establece el sistema europeo de créditos y el sistema de calificaciones en las titulaciones universitarias de carácter oficial y validez en todo el territorio nacional, indica sobre la incorporación del ECTS: "esta medida del haber académico comporta un nuevo modelo educativo que ha de orientar las programaciones y las metodologías docentes centrándolas en el aprendizaje de los estudiantes". En similar sentido se manifiestan Pagani y González (2002) cuando enfatizan en la necesidad de "una reorganización conceptual de los sistemas educativos para adaptarse a los nuevos modelos de formación centrados en el trabajo del estudiante" (p. 6).

Potenciar la enseñanza individualizada y que el centro del proceso sea el estudiante, supone modificar una enseñanza transmisiva a otra que permita construir significados y generar nuevos conocimientos, y tomar en consideración las diferencias individuales. Reisetter, Lapointe y Korcuska (2007) indican que en este tipo de enseñanza el alumno debe desplegar un importante trabajo individualizado, de manera autónoma, y que el éxito dependerá de su capacidad de autorregulación y de las percepciones positivas que tenga.

La adopción del ECTS implica reorganizar el currículo universitario, así como la forma de valorar el trabajo de los estudiantes, aunque esto es complejo en la docencia estrictamente presencial con la elevada ratio profesor/alumno que presenta la

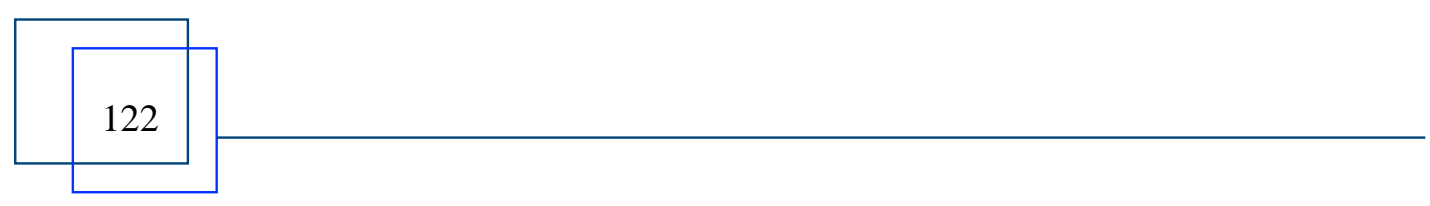




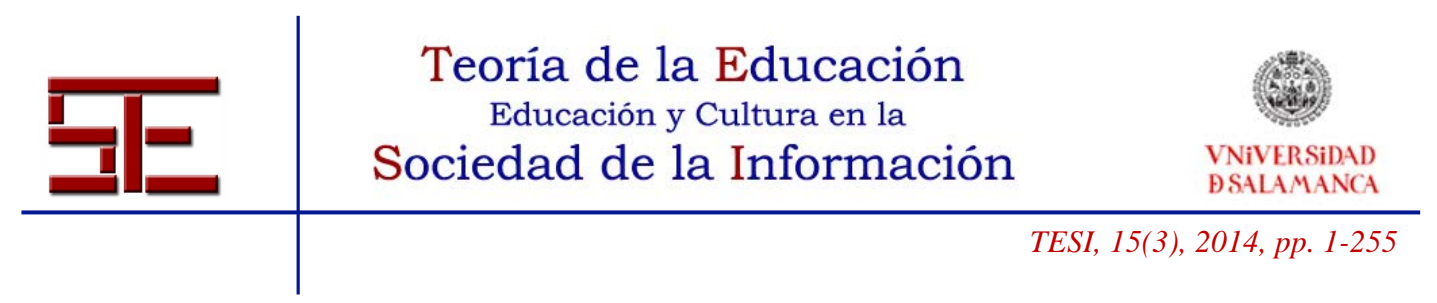

universidad española, en tanto que no permite un seguimiento minucioso del proceso de aprendizaje y el sistema de evaluación es básicamente sumativo. La incorporación de las plataformas educativas para mejorar los procesos de enseñanza-aprendizaje es una decidida apuesta para prestar una atención más individualizada a los estudiantes, a los que además se pone a su disposición una amplia gama de recursos de aprendizaje.

Tampoco se puede olvidar la importancia que actualmente presenta la formación en competencias de los estudiantes. En el caso de la asignatura de Materiales de Construcción-I, las competencias genéricas que se deben desarrollar son: capacidad para la resolución de problemas, capacidad para tomar decisiones, capacidad para el razonamiento crítico, capacidad de razonamiento, discusión y exposición de ideas propias, capacidad para el aprendizaje autónomo y desarrollar aquellas habilidades de aprendizaje necesarias para emprender estudios posteriores con un alto grado de autonomía. Entendemos que todas ellas se pueden abordar recurriendo a las TIC como un buen aliado. Cuando hablamos de autonomía de los estudiantes, estamos enfatizando en actividades que los docentes deben desplegar de manera importante para que los estudiantes alcancen sus competencias: la tutorización. Labor de vital importancia y que en este nuevo marco se redimensiona, en cuanto que el centro ya no es solo la adquisición de conocimientos por parte del estudiante y la resolución de dudas por el profesor, sino también la de acompañamiento y asesoramiento durante el proceso formativo para que el estudiante se desarrolle y crezca de manera integral.

Antes de continuar hemos de indicar que para los estudios de grado en la Universidad de Sevilla solo están permitidas las modalidades de docencia presencial y el blendedlearning, quedando el e-learning restringido a los estudios de postgrado y de formación permanente. En el caso que nos ocupa, estudios de grado, se permite, y se potencia, la incorporación de plataformas educativas como complemento a la formación presencial. Esta universidad optó por WebCT como recurso formativo.

\subsection{Blended learning y formación universitaria}

Aunque mucho se ha escrito acerca del blended-learning, entendemos que sigue siendo un término vigente y actual, en la medida que es una modalidad educativa que bien diseñada y planificada permite dar respuesta a las demandas universitarias actuales. Garrison y Vaughan (2008, p. 5) entienden que es "la fusión de la comunicación verbal de la enseñanza presencial y las experiencias de aprendizaje en línea. El principio básico es que la comunicación verbal de la enseñanza presencial y la escrita de la enseñanza en línea se integran de forma óptima, de manera que los puntos fuertes de cada uno se combinan en una experiencia de aprendizaje única, congruente con el contexto y el objetivo educativo".

El blended-learning se está incorporando como un modelo de enseñanza cada vez más

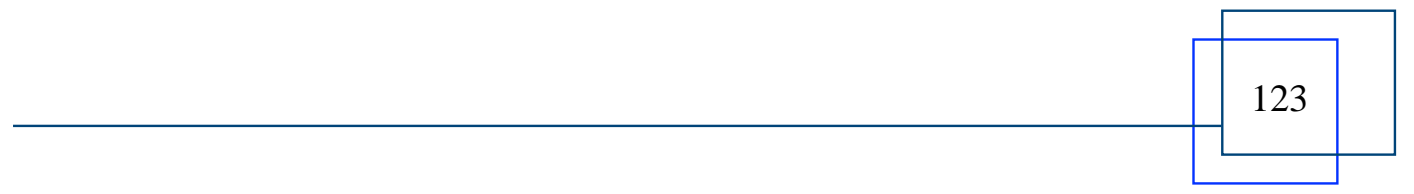




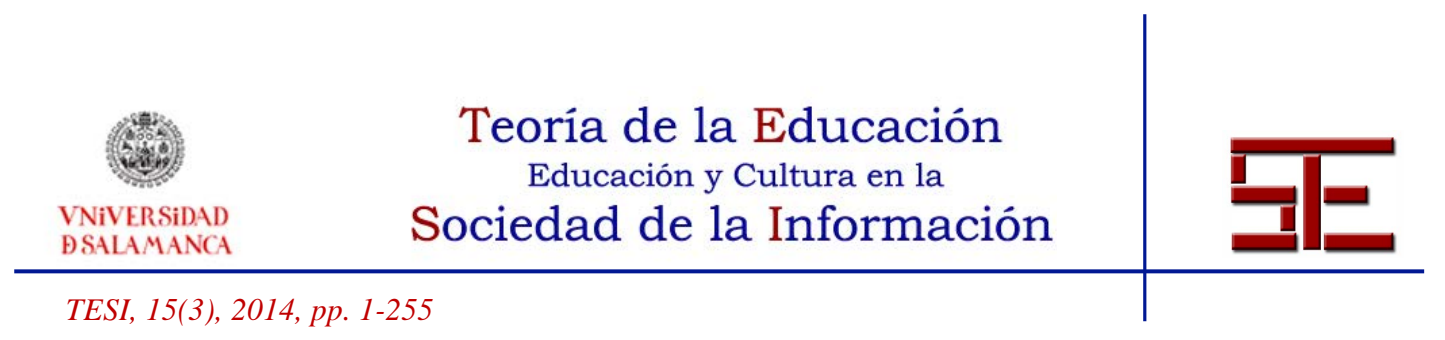

extendido en la enseñanza universitaria (Ellis, Ginns \& Piggott, 2009; MacKenzie \& Walsh, 2009), en el que se complementan los modelos de enseñanza presencial y online, permitiendo obtener lo mejor de ambos (Harding, Kaczynski \& Wood, 2005; Osguthorpe \& Graham, 2003). Y que entendemos da sobrada respuesta a los requerimientos expuestos de enseñanza flexible y centrada en el estudiante, creación de un entorno rico en recursos de aprendizaje, amplia posibilidad de asesoramiento y tutorización a través de las herramientas que las plataformas educativas incorporan, entre otros elementos de igual importancia.

Desde los objetivos que guían esta investigación, nos interesa destacar los siguientes aportes que hace el blended-learning a la enseñanza universitaria:

a) Posibilidad de crear nuevos espacios y escenarios comunicativos y de interacción (Ching \& Hsu, 2011; Neumann, Neumann, \& Hood, 2011; Brindley, Walti, \& Blaschke, 2009; Raban \& Litchfield, 2007). De la necesidad de presencialidad, en un proceso formativo tradicional con procesos comunicativos puramente síncronos, las TIC ofrecen no solo la posibilidad de sincronía sino también la comunicación asíncrona, flexibilizando los procesos comunicativos. Este nuevo escenario permite incrementar el grado de interacción entre los participantes en la acción formativa. Para Carr-Chellman, Dyer y Breman (2000) las ventajas fundamentales se encuentran en que el estudiante puede participar en cualquier momento y en cualquier lugar, y que el tiempo de clase le permite formar parte de una comunidad de aprendizaje. Pero además se debe destacar que la posibilidad de la comunicación asíncrona le ofrece al estudiante un tiempo de reflexión y creatividad (Shannon, Francis, Leng Chooi, \& Lynn, 2012; Means, Toyama, Murphy, Bakia, \& Jones, 2009; Mosca, Ball, Buzza, \& Paul, 2010) antes de ofrecer la respuesta demandada; reflexión que engloba la búsqueda de información, análisis de la misma, estructuración y publicación. En este sentido es fundamental la interacción que se produce entre estudiantes y docentes en los foros y chats. Y todo ello propiciado por la deslocalización física y temporal que emana del empleo de las plataformas educativas.

b) Nuevos escenarios de aprendizaje (Cooner, 2010; De Witt \& Kerr, 2003). En la enseñanza presencial la información a transformar en conocimiento por el estudiante es transmitida por el profesor, a través de las TIC el estudiante puede acceder a todo tipo de información y será él quien seleccione qué información quiere utilizar, lo que Cabero (2007a, p. 98) llama "deslocalizar la información de los contextos cercanos". Es de destacar el papel que puede, y debe, asumir el estudiante, como ya apuntábamos de constructor de su conocimiento, pero también como gestor de su tiempo (entorno flexible de aprendizaje), como indican Cabero (2007a, 2007b), Berlanga, Bosom y Hernández (2007), Acosta Lugo (2004), entre otros.

Pero además, entendemos que se deben reseñar las posibilidades que le presta al estudiante. En primer lugar, como individuo en un proceso de aprendizaje

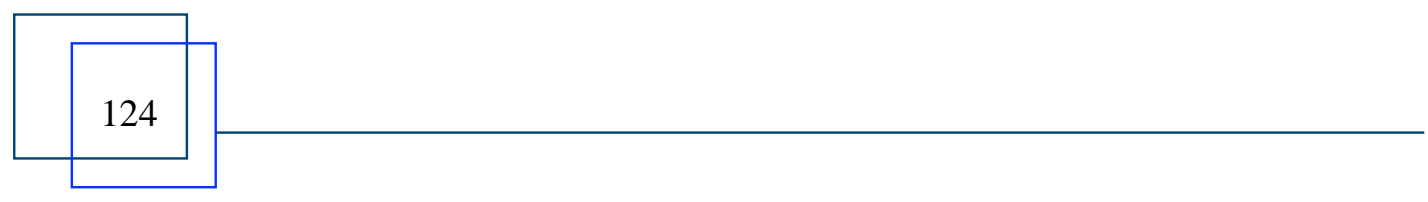




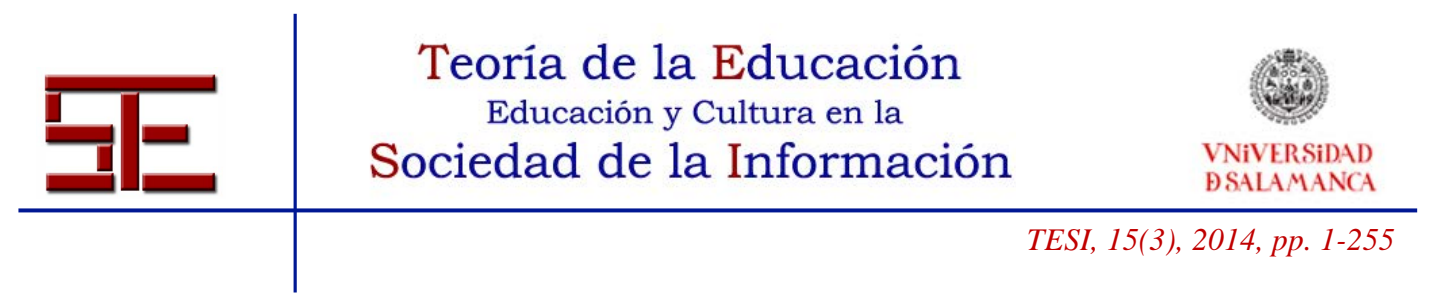

independiente; para ello, los materiales deberán estar estructurados de manera que lo permita y facilite. De otra parte, las altas prestaciones para el aprendizaje colaborativo a través de la interacción con el resto de miembros del curso, en la medida que al ser el conocimiento un proceso de construcción social, se ve claramente fortalecido a través de los medios tecnológicos disponibles. Como consecuencia de ello, aumentan las posibilidades para la orientación y tutorización (Cabero, 2007b; Berlanga et al., 2007). Sin olvidar que el disponer de estos recursos aumenta la flexibilidad para el desempeño académico (Neto, Vieira, Moreira, \& Ribeiro, 2013; Cheng \& Tsai, 2012; Davidson, 2011; Stricker, Weible, \& Bissmath, 2011), propicia un ambiente autónomo de aprendizaje (Meurant, 2010; Vázquez-Martínez \& Alducin-Ochoa, 2008) y mayor conocimiento del trabajo de los estudiantes (Vázquez-Martínez, 2011; Sitzmann, Kraiger, Stewart, \& Wisher, 2006).

Respecto a la investigación sobre el binomio blended-learning y enseñanzas técnicas, Hölb y Welzer (2010) indican que está aún en sus inicios. Pero afortunadamente se están dando pasos importantes (González Rogado, Rodríguez Conde, Olmos Migueláñez, García Riaza, García Peñalvo, 2010; González, Rodríguez, Olmos, Borham, García, 2013) no solo para cambiar la metodología docente, reflexionar sobre la práctica docente, sino también para la difusión de los resultados en materia TIC, concretamente en blended-learning, como así lo atestiguan los 43 artículos que sobre esta temática se han publicado durante el periodo 2006-2013 en revistas incluidas en WoS (Web of Science), 16 de los cuales son de investigadores españoles.

\subsection{Blended learning en la asignatura Materiales de Construcción-I}

El diseño de los componentes que forman parte de la plataforma de Materiales de Construcción-I estuvo basado en los objetivos y competencias que los estudiantes debían alcanzar y en las recomendaciones de Cabero y Gisbert (2005) para este tipo de producciones. Así como en los principios de: cuanto menos más, lo técnico supeditado a lo didáctico, legibilidad contra irritabilidad, evitar el aburrimiento, interactividad, hipertextualidad, flexibilidad (Cabero, 2002).

Los elementos de los que dispone el estudiante en WebCT los podemos agrupar en medios y recursos de aprendizaje y herramientas de comunicación. En el primer grupo se encuentran los contenidos y sus correspondientes elementos de apoyo, que hemos dado en llamar "Mi biblioteca". Y en las herramientas de comunicación, las bidireccionales (correo, foros y chats) y las monodireccionales (anuncios).

Los contenidos se engloban en torno a tres unidades didácticas (Rocas, Cerámica y Vidrios), desarrolladas en los correspondientes temas. Se desarrollan en formato HTML y se organizan siguiendo el criterio clásico: introducción histórica- materias primasprocesos de fabricación- procesos de transformación- estudio de propiedades- 


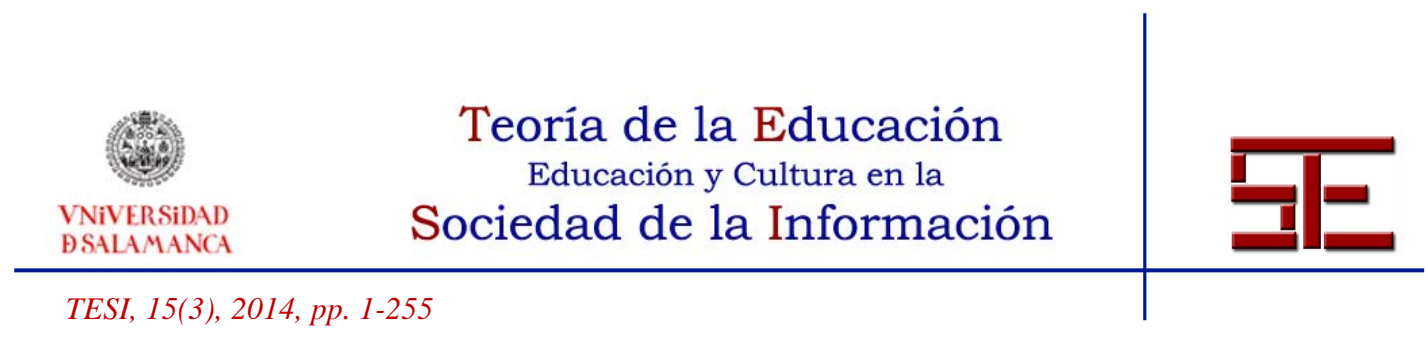

variedades de productos- patología. Al respecto se debe indicar que: el estudiante no puede imprimir los apuntes directamente, los debe elaborar él mismo, como quiera, como más le guste; cada tema dispone de un mapa conceptual por el que el estudiante puede moverse libremente; a cada tema están vinculados los objetivos; el estudiante dispone de marcadores para facilitar el estudio y de la opción de notas para gestionar mejor su proceso de aprendizaje; cada tema está vinculado a una categoría de discusión en el foro; la cantidad de contenido por pantalla se intenta que sea la menor posible, intentando fraccionar cuando es preciso en apartados y subapartados, antes que cargar demasiado un apartado concreto. Al final de cada tema se encuentran las pruebas de autorregulación de aprendizajes, como son las evaluaciones temáticas, y al final de la Unidad didáctica la evaluación de integración global de conocimientos y las e-tareas con sus correspondientes rúbricas de evaluación. Con estas concluye la preparación de cada Unidad, y pretenden dar un enfoque práctico a la asignatura (saber hacer), una vez que los alumnos tienen un dominio suficiente de los contenidos teóricos y clínicos (prácticas de laboratorios), la resolución exige diseñar un plan de acción, su desarrollo, determinar qué se precisa para su resolución, localizarlos, analizarlos, discriminar lo valioso, elaborar la respuesta y argumentarla.

Respecto a las pruebas de evaluación se debe indicar la tipología de las mismas:

Autoevaluación: pruebas con las que se intenta centrar la atención del estudiante, obligarle a la lectura pormenorizada del tema, que sea capaz de distinguir las ideas principales, así como identificar aquellos elementos que presentan dificultades de comprensión.

De liberación: organizadas por tres niveles de dificultad. Le permiten al estudiante el acceso al tema siguiente y a la prueba de evaluación del tema sometido a estudio. Debemos indicar que el estudiante solo tiene en abierto el primer tema de cada Unidad Didáctica, el resto los debe ir abriendo al demostrar un nivel de conocimiento mínimo en los temas anteriores.

De evaluación: pruebas de mayor nivel de dificultad que las de liberación, y que sitúan al estudiante en su nivel real de conocimiento.

"Mi biblioteca" está organizada por Unidades didácticas y subclasificadas siguiendo el mapa conceptual de cada tema. El estudiante encuentra en ella 104 referencias bibliográficas sin acceso directo pero disponibles en las bibliotecas de la universidad de Sevilla; 75 vídeos de acceso directo, 325 artículos en línea y 75 enlaces web. Estos recursos intentan promover la curiosidad del estudiante, y ser en primera instancia su banco de recursos para la resolución de las e-tareas.

Respecto a las herramientas de comunicación nos centraremos en los foros, dado que es el elemento que se analiza por el interés que tiene conocer el grado de comunicación

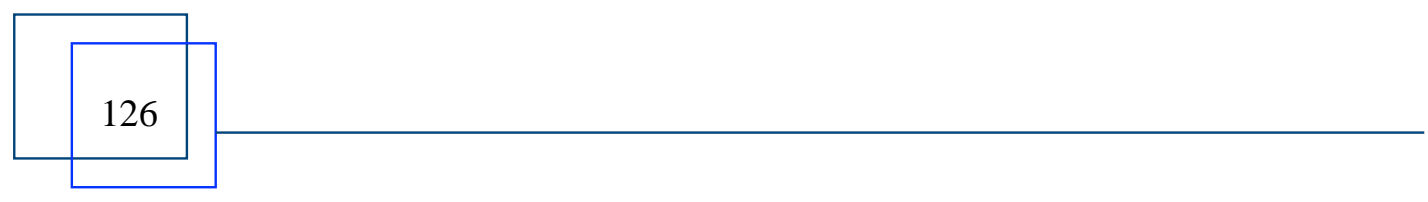




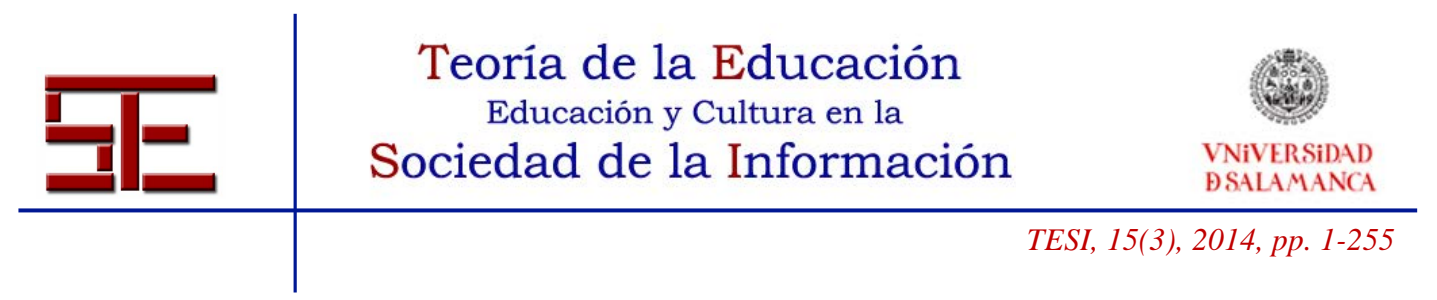

que se establece entre usuarios en este tipo de enseñanza. Tienen la función de facilitar la comunicación entre personas que están distantes en el espacio y en el tiempo. Aunque nuestros estudiantes están adscritos a un modelo de enseñanza presencial, no podemos olvidar que muchos de ellos no residen en zonas próximas a la universidad, y que en bastantes ocasiones los horarios de tutoría son incompatibles con la asistencia a clase. Y de otra parte la dedicación del profesorado a las tutorías presenciales, circunscritas a unos días y horas concretos, no siempre en relación con la aparición de la duda.

Por ello la implementación del foro en los sistemas de aprendizaje en línea propicia que los estudiantes puedan resolver las dudas que les impide continuar su proceso de aprendizaje en un breve periodo de tiempo, con independencia del espacio temporal en el que esta surja. Es por ello que el foro adquiere una singular importancia en el proceso de enseñanza - aprendizaje.

El foro de la asignatura Materiales de Construcción-I, está organizado en un primer momento en los dos grandes bloques que la forman: Problemas y Teoría, pero al mismo tiempo para facilitar que el estudiante sepa dónde puede ubicar su duda y posteriormente la localización de preguntas-respuestas por bloques temáticos, actividades y e-tareas encargadas a los estudiantes. La filosofía de funcionamiento ha sido potenciar al máximo que las respuestas sean dadas por los propios estudiantes y todo el proceso controlado por el profesor responsable de cada área de trabajo. El objetivo pretendido además de facilitar un aprendizaje de calidad, era desarrollar una metodología de aprendizaje colaborativo. Cuando un estudiante envía una duda al correo de los profesores que se entiende pueden presentar otros estudiantes, estos le invitan a plantearla a través del foro. Debemos indicar que durante el periodo de tiempo analizado, un cuatrimestre, se contabilizaron 1.935 mensajes, generados tanto por los alumnos participantes como por los profesores, estos emitieron 475 mensajes.

\section{METODOLOGÍA}

\subsection{Diseño}

Los objetivos de investigación planteados sugieren un diseño metodológico transversal, ex post facto con variables no manipuladas experimentalmente, empleando el método descriptivo y correlacional.

\subsection{Objetivos e hipótesis}

Los objetivos que guían esta investigación son:

1. Verificar la influencia del grado de empleo de las herramientas foro y

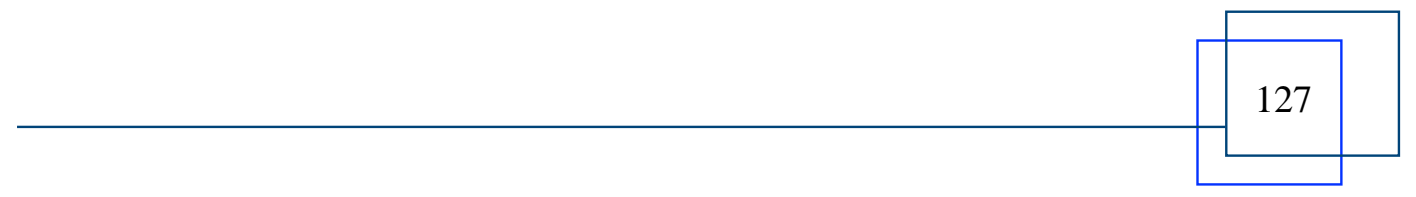




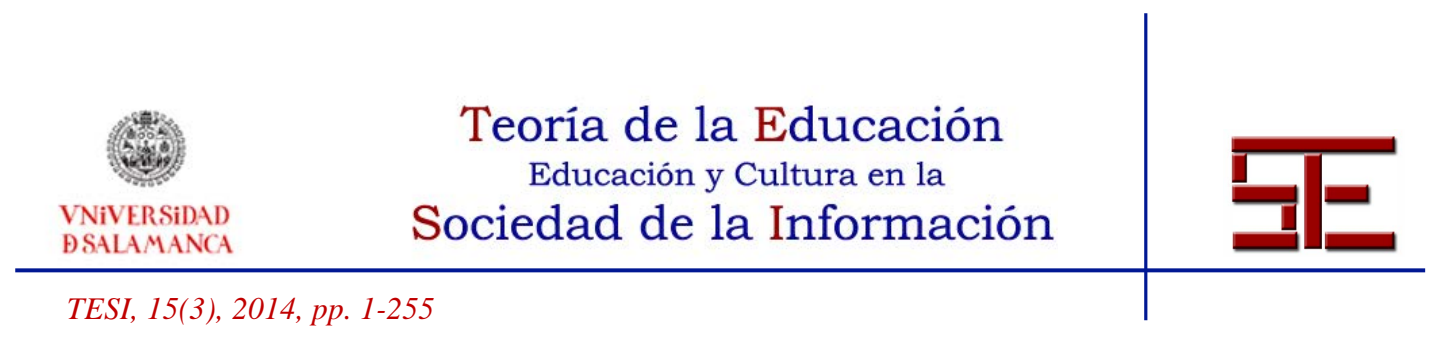

autoevaluación de WebCT por parte de los estudiantes en su rendimiento académico.

2. Comprobar la relación entre la valoración que realizan los estudiantes de WebCT y su rendimiento académico.

3. Comprobar la relación entre el grado de empleo de las herramientas foro y autoevaluación de WebCT y la valoración de la misma.

Y las hipótesis formuladas como hipótesis nulas:

- H1 $1_{0}$ : "No existen diferencias significativas en el rendimiento de los estudiantes en la asignatura Materiales de Construcción-I en función del grado de empleo de WebCT".

- H2 $2_{0}$ : "No existe correlación entre la valoración que realizan los estudiantes de WebCT y su rendimiento académico".

- H3 $3_{0}$ : "No existen diferencias significativas en la percepción y satisfacción del empleo de WebCT en función del grado de empleo de la misma".

\subsection{Participantes}

La muestra para el estudio estuvo constituida por los 176 estudiantes que comenzaron el curso, y que estaban adscritos a cuatro de los diez grupos en los que se imparte la asignatura, de los cuales 60 (34.09\%) son mujeres y 116 (65.91\%) hombres. Por edad, la distribución es 110 (62.50\%) estudiantes entre 18-20 años, 40 (22.73\%) entre 21-25 años, 17 (9.66\%) entre 26-30 años, y $9(5.11 \%)$ mayores de 30 años, siendo la media de 21.25 años. De ellos se dispone las calificaciones de las unidades didácticas. El Cuestionario de Percepción y Satisfacción del Empleo de WebCT (PSEW) fue cumplimentado por 116 estudiantes.

\subsection{Instrumentos}

Los datos de empleo de WebCT se obtuvieron de la opción "Seguimiento" de la propia plataforma. Y los referentes a su valoración con el cuestionario de Percepción y Satisfacción del Empleo de WebCT (PSEW) diseñado al efecto con 48 ítems escala de tipo Likert distribuidos en cuatro dimensiones. Se determinó la fiabilidad del cuestionario PSEW empleándose el coeficiente alfa de Cronbach, obteniéndose $\alpha=.923$ que indica, según George y Mallery (1995), un nivel excelente de fiabilidad.

\subsection{Análisis de los datos}

Se ha empleado el paquete informático SPSS versión 17. Para los objetivos 1 y 3 se

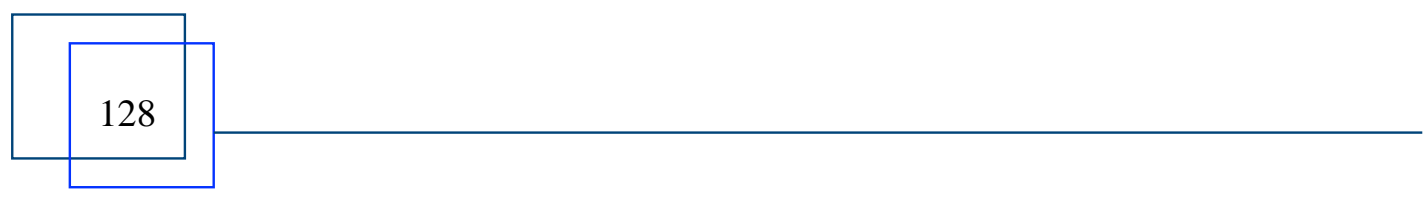




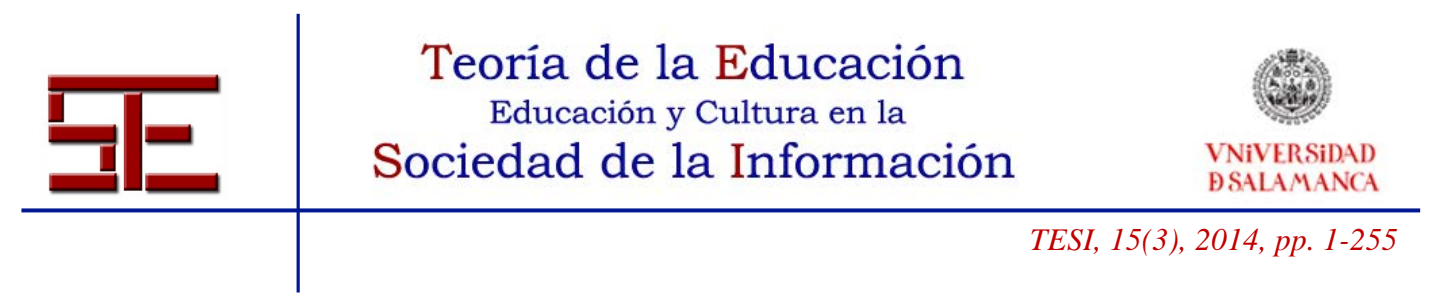

emplearán pruebas paramétricas cuando la variable dependiente esté medida a nivel de intervalo y tenga una distribución normal, concretamente la prueba $\mathrm{T}$ de student si la variable independiente es dicotómica o el análisis de la varianza (ANOVA) cuando la variable independiente sea politómica; y se emplearán pruebas no paramétricas cuando la variable dependiente no alcance el nivel de medida de intervalo o no siga la distribución normal, específicamente la prueba U de Mann-Whitney si la variable independiente es dicotómica o la prueba de Kruskal-Wallis cuando la variable independiente sea politómica. Para el objetivo 2 se empleará el coeficiente de correlación Rho de Spearman. En todas las pruebas se establece un nivel de confianza del $95 \%(\alpha=.05)$.

\section{RESULTADOS}

\subsection{Influencia del empleo de WebCT en el rendimiento}

Para analizar la posible influencia del grado de empleo de las pruebas preparatorias en el rendimiento, se establecen tres grupos de empleo a partir del número de pruebas de autoevaluación realizadas por los estudiantes: inferior, número de pruebas realizadas entre 1 y percentil 33; intermedio, número de pruebas realizadas entre percentil 33 y percentil 67; superior, número de pruebas realizadas entre percentil $67 \mathrm{y}$ el valor máximo de pruebas realizadas.

En las calificaciones de la Unidad 2, la prueba de normalidad de Kolmogorov-Smirnov (tabla 1) indica que no se sigue la distribución normal al haber alguna categoría de la variable de agrupación "grado de empleo de las autoevaluaciones" con $\mathrm{p}<.05$. Mientras que en las calificaciones de las Unidades 1 y 3 sí se cumple el supuesto de normalidad al tener en las tres categorías del grado de empleo de las autoevaluaciones valores de $\mathrm{p}>.05$.

Tabla 1. Prueba de normalidad de las calificaciones por empleo de las autoevaluaciones.

\begin{tabular}{llllll}
\hline & & \multicolumn{4}{c}{ Kolmogorov-Smirnov } \\
Calificaciones & $\begin{array}{l}\text { Grado empleo } \\
\text { autoevaluaciones }\end{array}$ & Estadístico & $\mathrm{gl}$ & Sig. \\
\hline Unidad didáctica 1 & Inferior & .104 & 41 & $.200^{*}$ \\
& Intermedio & .080 & 61 & $.200^{*}$ \\
& Superior & .104 & 68 & .065 \\
Unidad didáctica 2 & Inferior & .130 & 27 & $.200^{*}$
\end{tabular}




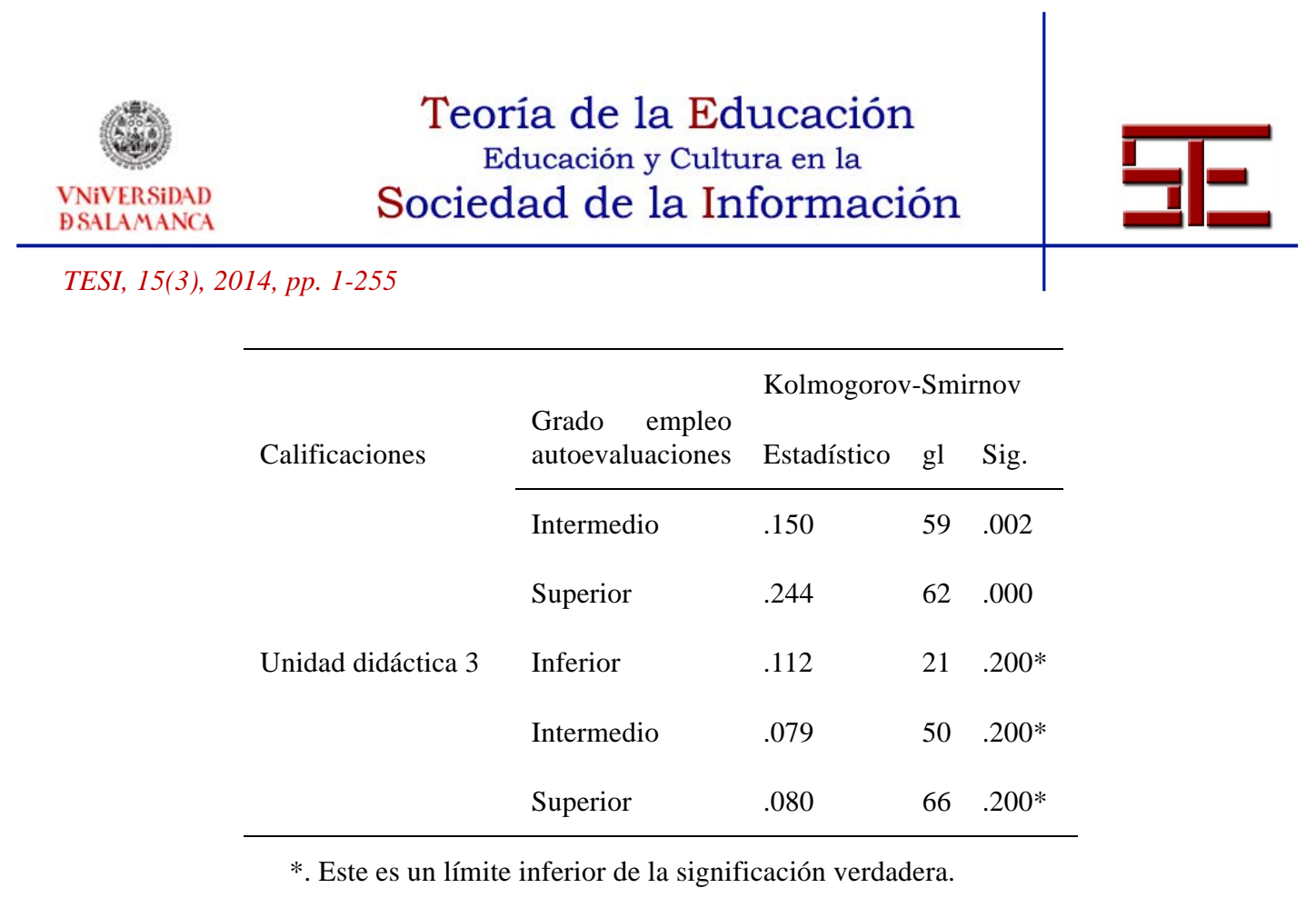

La tabla 2 recoge las medias de las notas obtenidas por los estudiantes en las tres unidades, en función del grado de empleo de las pruebas de autoevaluación, y los rangos promedios de aquellas unidades en las que al no cumplirse el supuesto de normalidad se empleará la prueba de Kruskal-Wallis.

Tabla 2. Descriptivos de calificaciones en unidades didácticas por grado empleo de autoevaluaciones.

\begin{tabular}{lllllll}
\hline \multirow{2}{*}{ Nota } & $\begin{array}{l}\text { Grado empleo } \\
\text { autoevaluación }\end{array}$ & $\mathrm{N}$ & $\mathrm{M}$ & $\mathrm{SD}$ & $\begin{array}{l}\text { Promedio (RP) } \\
\end{array}$ \\
& & 41 & 3.33 & 2.178 & \\
\hline Unidad didáctica 1 & Inferior & 61 & 4.71 & 2.054 & \\
& Intermedio & & & & \\
& Superior & 68 & 5.88 & 1.631 & \\
Unidad didáctica 2 & Inferior & 27 & 3.21 & 2.061 & 35.31 \\
& Intermedio & 59 & 5.06 & 2.296 & 67.91 \\
& Superior & 62 & 6.71 & 1.298 & 97.84 \\
& Inferior & 21 & 4.13 & 1.720 & \\
Unidad didáctica 3 & Intermedio & 50 & 4.61 & 2.034 & \\
& Superior & 66 & 5.20 & 1.548 & \\
\hline
\end{tabular}

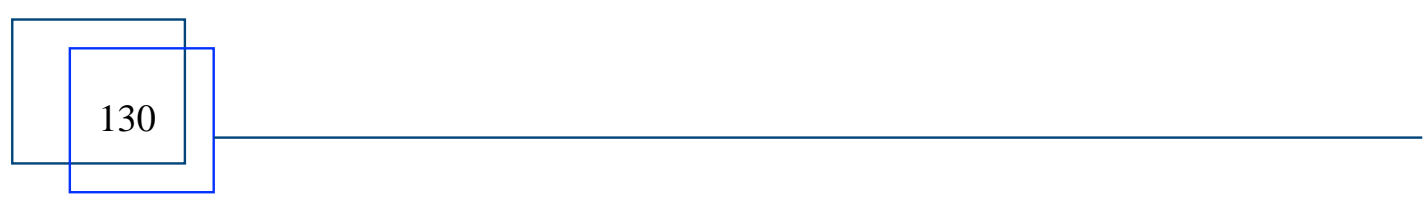




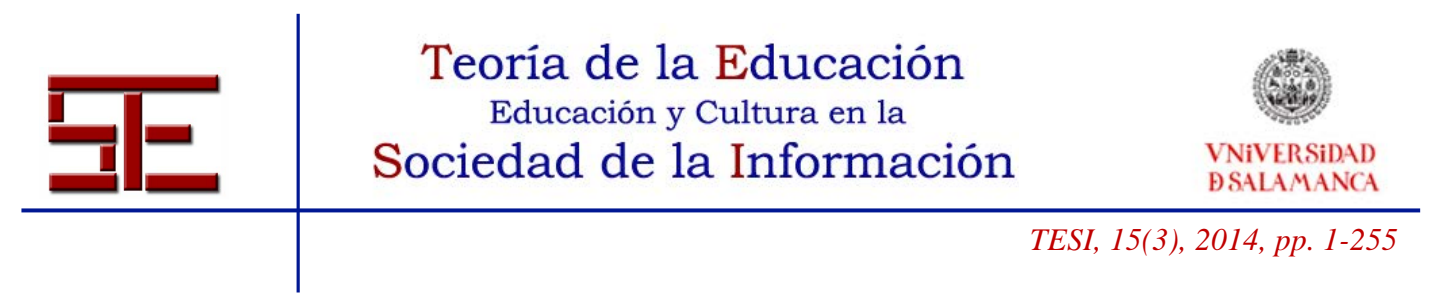

El cumplimiento del supuesto de normalidad en las calificaciones de las Unidades 1 y 3 permite emplear el análisis de la varianza (ANOVA) (tabla 3), mientras que para las calificaciones de la unidad 2 se empleará la prueba no paramétrica de Kruskal-Wallis (tabla 4).

Tabla 3. ANOVA para calificaciones agrupadas por grado de empleo de las autoevaluaciones.

\begin{tabular}{lllllll} 
& $\begin{array}{l}\text { Prueba de } \\
\text { Levene }\end{array}$ & \multicolumn{5}{c}{ ANOVA } \\
& Estadístico & Sig. & F & gl1 & gl2 & Sig. \\
\hline Ud. didác. 1 & 2.663 & .073 & 22.728 & 2 & 167 & .000 \\
Ud. didác. 3 & 2.049 & .133 & 3.470 & 2 & 134 & .034 \\
\hline
\end{tabular}

La prueba de Levene en el ANOVA de las calificaciones de las Unidades 1 y 3 ofrece una significación $\mathrm{p}=.073, \mathrm{p}=.133$ respectivamente, que al ser mayores que .05 indica que se cumple el supuesto de homocedasticidad, por lo que empleamos la F de Snedecor del ANOVA. Se obtiene que existen diferencias significativas en:

- las calificaciones de la Unidad 1 entre la media del grado inferior de empleo de las pruebas $(\mathrm{M}=3.33 ; \mathrm{SD}=2.178)$, del grado intermedio $(\mathrm{M}=4.71 ; \mathrm{SD}=$ 2.054) y del grado superior $(\mathrm{M}=5.88 ; \mathrm{SD}=1.631), \mathrm{F}(2,167)=22.728, \mathrm{p}=$ .000 , siendo la prueba post hoc HSD de Tukey la que confirma que la significatividad se debe a las diferencias entre el grado inferior e intermedio $(\mathrm{p}=$ $.001)$, inferior y superior $(\mathrm{p}=.000)$ y entre el grado intermedio y superior $(\mathrm{p}=$ $.002)$

- las calificaciones de la Unidad 3 entre la media del grado inferior de empleo de las pruebas $(\mathrm{M}=4.13 ; \mathrm{SD}=1.720)$, del grado intermedio $(\mathrm{M}=4.61 ; \mathrm{SD}=$ 2.034) y del grado superior $(\mathrm{M}=5.20 ; \mathrm{SD}=1.548), \mathrm{F}(2,134)=3.470, \mathrm{p}=.034$, siendo la prueba post hoc HSD de Tukey la que confirma que la significatividad se debe a las diferencias entre el grado inferior y superior $(\mathrm{p}=.045)$.

Tabla 4. Prueba Kruskal-Wallis para calificaciones agrupadas por grado empleo de las autoevaluaciones.

\begin{tabular}{lllll}
\hline & \multicolumn{4}{c}{ Kruskal-Wallis Test } \\
& $\mathrm{N}$ & Chi-cuadrado & gl & Sig. \\
\hline Unidad didáctica 2 & 148 & 42.336 & 2 & .000 \\
\hline
\end{tabular}

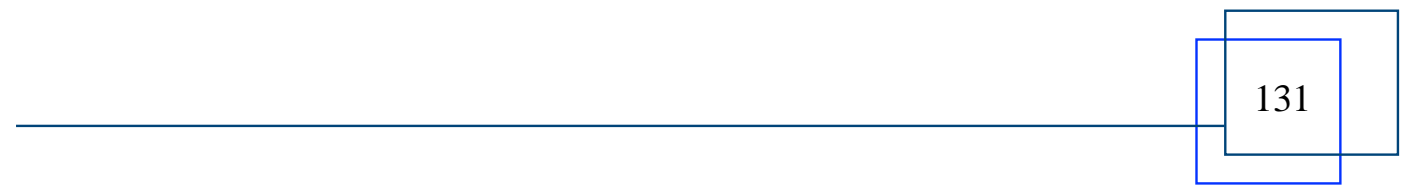




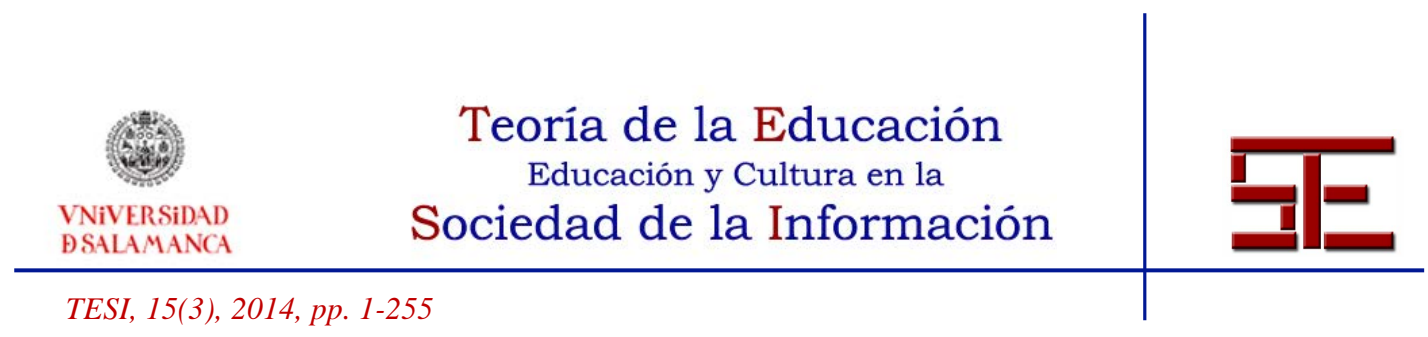

La prueba no paramétrica de Kruskal-Wallis indica que se encontraron diferencias significativas en las calificaciones de la Unidad 2 entre los rangos promedio del grado inferior $(\mathrm{RP}=35.31)$, del grado intermedio $(\mathrm{RP}=67.91)$ y del grado superior $(\mathrm{RP}=$ 97.84), $\mathrm{X}^{2}(2, \mathrm{~N}=148)=42.336, \mathrm{p}=.000$. Por ello, se acepta la hipótesis alternativa "Existen diferencias significativas en el rendimiento de los estudiantes en la asignatura Materiales de Construcción-I por el grado de empleo de las pruebas de autoevaluación en las tres unidades didácticas".

Para analizar la posible influencia del grado de empleo del foro en el rendimiento, se establecen tres grupos de empleo a partir del número de mensajes escritos en el foro: inferior, número de mensajes entre 1 y percentil 33; intermedio, número de mensajes entre percentil 33 y percentil 67; superior, número de mensajes entre percentil 67 y el valor máximo de mensajes.

La prueba de normalidad de Kolmogorov-Smirnov (tabla 5) indica que no se sigue la distribución normal en las calificaciones de la Unidades 1 y 2, mientras que las de la Unidad 3 sí se cumplen los supuestos de normalidad.

Tabla 5. Prueba de normalidad de las calificaciones por grado de empleo del foro.

\begin{tabular}{llllll}
\hline & & \multicolumn{3}{c}{ Kolmogorov-Smirnov } \\
Calificaciones & Grado empleo & del foro & Estadístico & $\mathrm{gl}$ & Sig. \\
\hline Unidad didáctica 1 & Inferior & .130 & 30 & $.200^{*}$ \\
& Intermedio & .109 & 30 & $.200^{*}$ \\
& Superior & .187 & 32 & .006 \\
Unidad didáctica 2 & Inferior & .122 & 26 & $.200^{*}$ \\
& Intermedio & .315 & 28 & .000 \\
& Superior & .289 & 32 & .000 \\
& Inferior & .135 & 24 & $.200^{*}$ \\
& Intermedio & .124 & 28 & $.200^{*}$ \\
& Superior & .127 & 32 & $.200^{*}$ \\
\hline
\end{tabular}

* Este es un límite inferior de la significación verdadera.

La tabla 6 recoge las medias de las notas obtenidas por los estudiantes en las tres

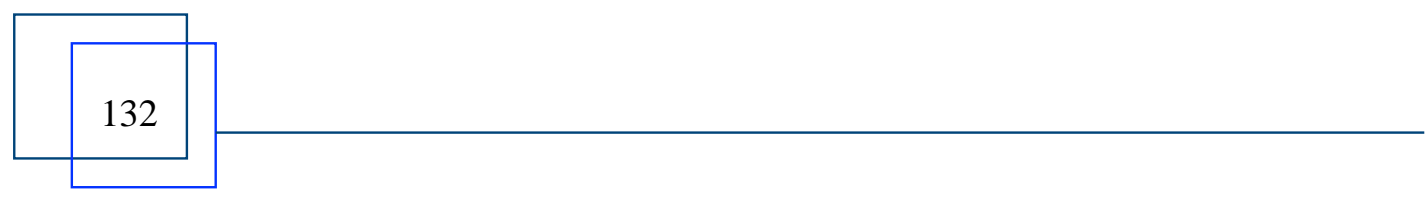




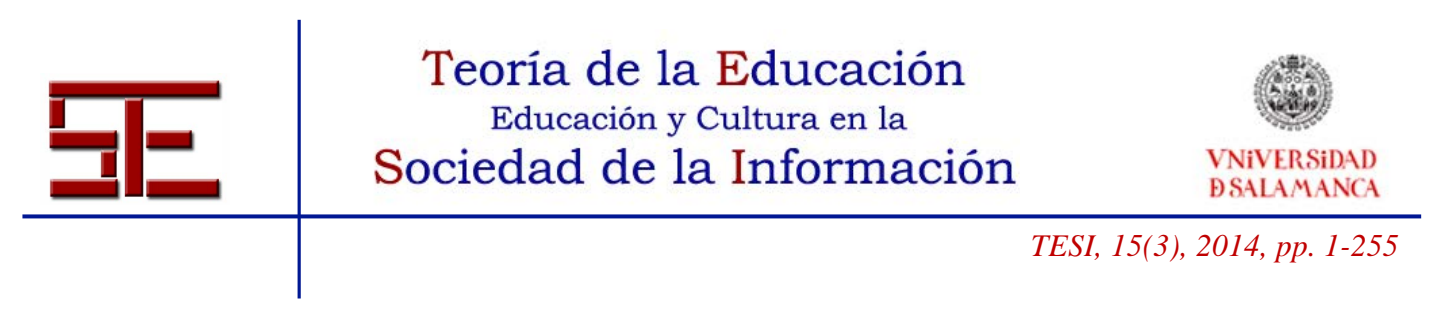

unidades, en función del grado de empleo del foro, y los rangos promedios de aquellas unidades en las que al no cumplirse el supuesto de normalidad se empleará la prueba de Kruskal-Wallis.

Tabla 6. Descriptivos de calificaciones en unidades didácticas por grado empleo del foro.

\begin{tabular}{|c|c|c|c|c|c|}
\hline \multirow{3}{*}{ Calificaciones } & \multirow{3}{*}{$\begin{array}{l}\text { Grado empleo } \\
\text { del foro }\end{array}$} & \multicolumn{3}{|c|}{ Descriptivos } & \multirow[b]{2}{*}{ Rangos } \\
\hline & & & & & \\
\hline & & $\mathrm{N}$ & M & SD & Promedio (RP) \\
\hline \multirow[t]{3}{*}{ Unidad didáctica 1} & Inferior & 30 & 4.59 & 2.198 & 38.72 \\
\hline & Intermedio & 30 & 4.70 & 1.937 & 38.87 \\
\hline & Superior & 32 & 6.27 & 1.829 & 60.95 \\
\hline \multirow[t]{3}{*}{ Unidad didáctica 2} & Inferior & 26 & 4.94 & 1.952 & 30.77 \\
\hline & Intermedio & 28 & 6.21 & 1.935 & 47.89 \\
\hline & Superior & 32 & 6.33 & 1.808 & 50.00 \\
\hline \multirow[t]{3}{*}{ Unidad didáctica 3} & Inferior & 24 & 4.45 & 1.713 & \\
\hline & Intermedio & 28 & 4.52 & 2.127 & \\
\hline & Superior & 32 & 5.53 & 1.411 & \\
\hline
\end{tabular}

El cumplimiento del supuesto de normalidad en las calificaciones de la Unidad 3 permite emplear el análisis de la varianza (ANOVA) (tabla 7), mientras que para las calificaciones de las unidades 1 y 2 se empleará la prueba no paramétrica de KruskalWallis, dado que no se cumple el supuesto de normalidad (tabla 8).

Tabla 7. ANOVA para calificaciones agrupadas por grado de empleo del foro.

\begin{tabular}{|c|c|c|c|c|c|c|}
\hline & \multicolumn{2}{|c|}{ Prueba de Levene } & \multicolumn{4}{|c|}{$\begin{array}{l}\text { ANOVA } \\
\text { Snedecor }\end{array}$} \\
\hline & Estadístico & Sig. & F & $\begin{array}{l}\mathrm{gl} \\
1\end{array}$ & $\mathrm{gl} 2$ & Sig. \\
\hline Ud. didác. 3 & 3.004 & .055 & 3.517 & 2 & 81 & .034 \\
\hline
\end{tabular}

La prueba de Levene en el ANOVA de las calificaciones de la Unidad 3 ofrece una

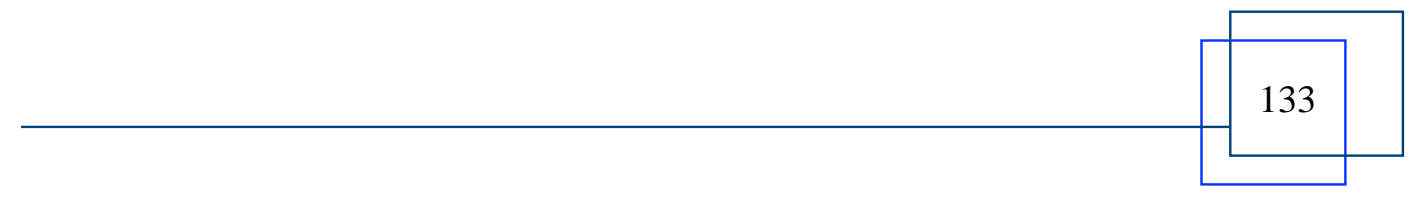




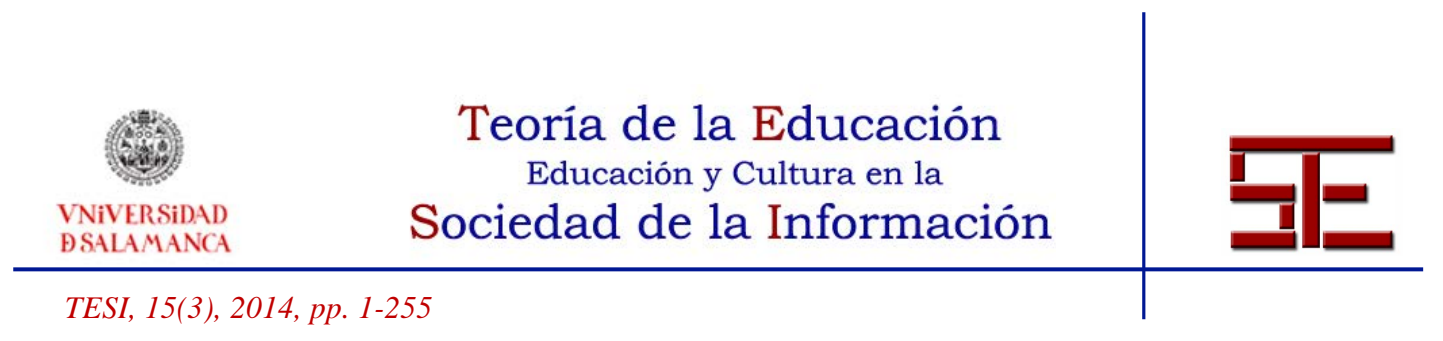

significación $\mathrm{p}=.055$, que al ser mayor que .05 indica que se cumple el supuesto de homocedasticidad, por lo que empleamos la F de Snedecor del ANOVA. Se obtiene que existen diferencias significativas en las calificaciones de la Unidad 3 entre la media del grado inferior de empleo del foro $(\mathrm{M}=4.45 ; \mathrm{SD}=1.713)$, del grado intermedio $(\mathrm{M}=$ $4.52 ; \mathrm{SD}=2.127)$ y del grado superior $(\mathrm{M}=5.53 ; \mathrm{SD}=1.411), \mathrm{F}(2,81)=3.517, \mathrm{p}=$ .034 , siendo la prueba post hoc DMS la que confirma que la significatividad se debe a las diferencias entre el grado inferior y superior $(\mathrm{p}=.025)$, e intermedio y superior $(\mathrm{p}=$ $.029)$.

Tabla 8. Prueba Kruskal-Wallis para calificaciones agrupadas por grado de empleo del foro.

\begin{tabular}{lllll}
\hline & \multicolumn{5}{c}{ Kruskal-Wallis Test } \\
& $\mathrm{N}$ & Chi-cuadrado & $\mathrm{gl}$ & $\mathrm{S}$ Sig. \\
\hline Unidad didáctica 1 & 92 & 14.383 & 2 & .001 \\
Unidad didáctica 2 & 86 & 9.795 & 2 & .007 \\
\hline
\end{tabular}

La prueba no paramétrica de Kruskal-Wallis indica que se encontraron diferencias significativas en:

- las calificaciones de la Unidad 1 entre los rangos promedio del grado inferior de empleo del foro $(\mathrm{RP}=38.72)$, del grado intermedio $(\mathrm{RP}=38.87)$ y del grado superior $(R P=60.95), X^{2}(2, N=92)=14.383, p=.001$.

- las calificaciones de la Unidad 2 entre los rangos promedio del grado inferior $(\mathrm{RP}=30.77)$, del grado intermedio $(\mathrm{RP}=47.89) \mathrm{y}$ del grado superior $(\mathrm{RP}=$ 50.00), $\mathrm{X}^{2}(2, \mathrm{~N}=86)=9.795, \mathrm{p}=.007$.

Por ello, se acepta la hipótesis alternativa "Existen diferencias significativas en el rendimiento de los estudiantes en la asignatura Materiales de Construcción-I por el grado de empleo del foro en las tres unidades didácticas".

En el gráfico 1 se recogen las calificaciones medias obtenidas por los estudiantes, en función del grado de empleo de las pruebas de autoevaluación disponibles y de mensajes publicados en el foro.

Grafico 1: Calificaciones medias en función del grado de empleo de pruebas de autoevaluación y foro.

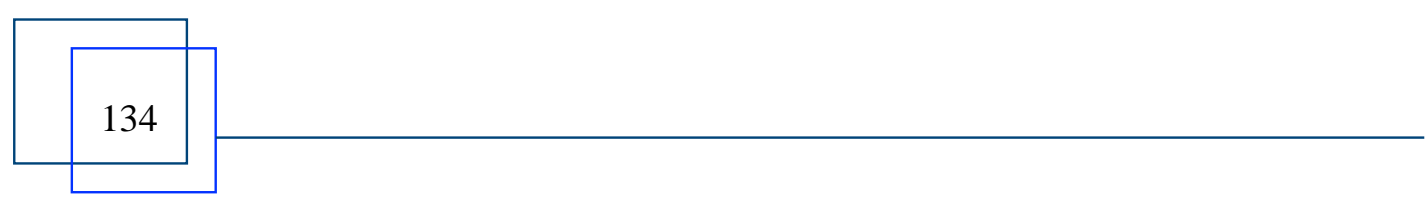



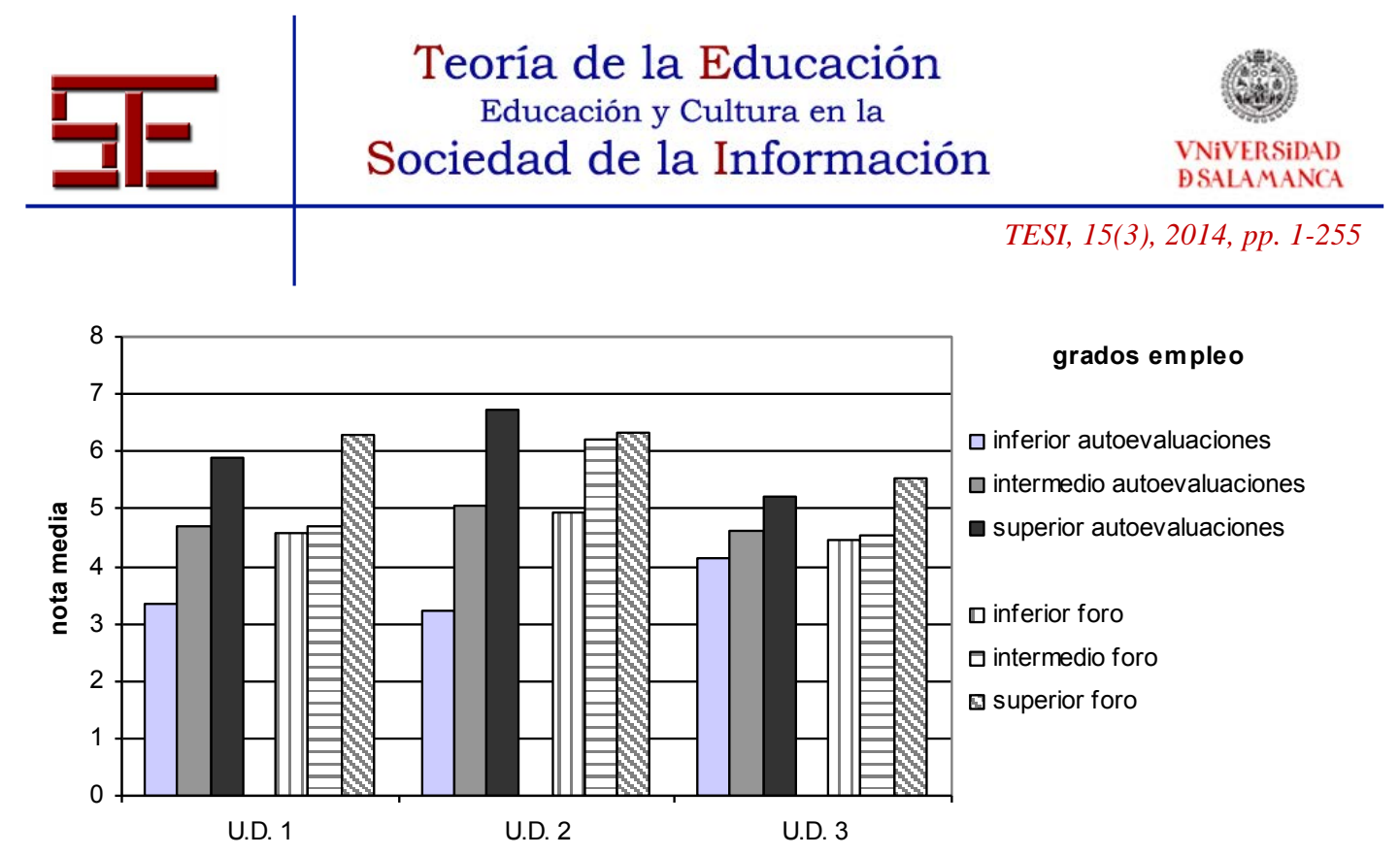

\subsection{Relación entre la valoración de WebCT y el rendimiento}

Para determinar si hay relación entre la valoración de Web-CT y el rendimiento académico se recurre a un estudio correlacional. Como las variables de valoración de la plataforma son cuantitativas con un nivel de medida ordinal, por proceder del cuestionario PSEW que emplea una escala de tipo Likert, y las variables de las calificaciones son cuantitativas con un nivel de medida de intervalo, se emplea el coeficiente de correlación Rho de Spearman. En la tabla 9 se recoge los coeficientes de correlación para los posibles binomios de estudio entre la valoración de WebCT y la calificación media de las tres unidades didácticas.

Tabla 9. Coeficientes de correlación entre valoración de WebCT y rendimiento académico.

\begin{tabular}{lllll}
\hline & \multicolumn{3}{c}{ Rho de Spearman } \\
Valoración WebCT & $\mathrm{N}$ & Coeficiente & Sig. \\
\hline Dimensión contenidos & 116 & $.322^{* *}$ & .000 \\
$\begin{array}{l}\text { Dimensión aspectos formales } \\
\text { técnicos }\end{array}$ & y & 116 & $.208^{*}$ & .025 \\
$\begin{array}{l}\text { Dimensión pruebas autoevaluación } \\
\text { Valoración global }\end{array}$ & 116 & $.501 * *$ & .000 \\
\hline
\end{tabular}

**. La correlación es significativa al nivel 0.01 (bilateral).

*. La correlación es significativa al nivel 0.05 (bilateral). 


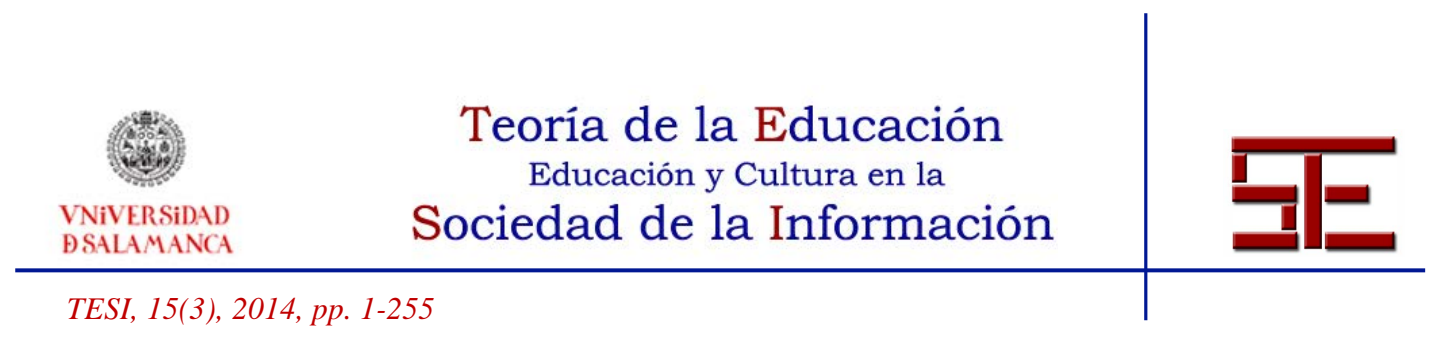

Al analizar los resultados de los coeficientes de correlación de Spearman entre las cuatro dimensiones de la valoración de WebCT con el rendimiento académico en el primer cuatrimestre, se obtienen los siguientes resultados:

- El coeficiente entre la valoración de la dimensión contenidos de WebCT y la nota del primer parcial, encontró correlación positiva estadísticamente significativa, $\mathrm{r}_{\mathrm{S}}(\mathrm{N}=116)=.322 ; \mathrm{p}=.000$ con un tamaño de efecto medio.

- El coeficiente entre la valoración de la dimensión aspectos formales y técnicos de WebCT y la nota del primer parcial, encontró correlación positiva estadísticamente significativa, $\mathrm{r}_{\mathrm{S}}(\mathrm{N}=116)=.208 ; \mathrm{p}=.025$ con un tamaño de efecto pequeño.

- El coeficiente entre la valoración de la dimensión pruebas de autoevaluación de WebCT y la nota del primer parcial, encontró correlación positiva estadísticamente significativa, $\mathrm{r}_{\mathrm{S}}(\mathrm{N}=116)=.501 ; \mathrm{p}=.000$ con un tamaño de efecto medio.

- El coeficiente entre la valoración global de WebCT y la nota del primer parcial, encontró correlación positiva estadísticamente significativa, $\mathrm{r}_{\mathrm{S}}(\mathrm{N}=116)=.215$; $\mathrm{p}=.020$ con un tamaño de efecto pequeño.

Los coeficientes de correlación confirman nuestra hipótesis, en consecuencia, se acepta la hipótesis alternativa "Existe correlación entre la valoración que realizan los estudiantes de WebCT y su rendimiento académico en las unidades didácticas con metodología docente basada en el empleo de WebCT".

\subsection{Relación entre el grado de empleo de la plataforma y la valoración de la misma}

Se pretende determinar si hay diferencias significativas en las medias de las valoraciones de percepción y satisfacción de empleo realizadas por los estudiantes, en función del grado de empleo de la plataforma. Para ello emplearemos una prueba no paramétrica al tener las variables de valoración de la plataforma un nivel de medida ordinal por proceder del cuestionario PSEW que emplea una escala de tipo Likert, concretamente se emplea la prueba de Kruskal-Wallis al tener las variables de agrupación referentes al grado de empleo de la plataforma tres categorías: inferior, intermedio y superior.

La tabla 10 recoge las medias y los rangos medios de las valoraciones por dimensiones de WebCT realizada por los estudiantes en función del grado de empleo de las pruebas de autoevaluación, y en la tabla 11 el resultado de la prueba de Kruskal-Wallis.

Tabla 10. Descriptivos de la valoración de WebCT por grado empleo de autoevaluaciones.

136 


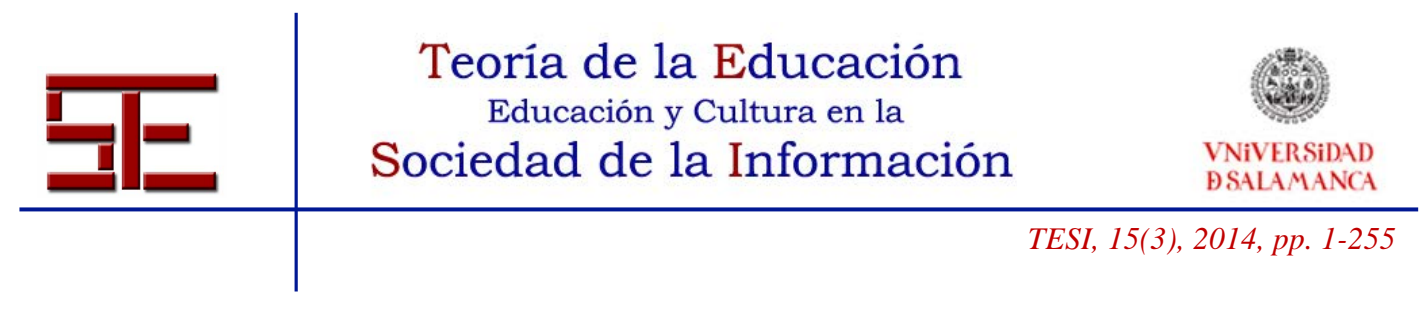

\begin{tabular}{|c|c|c|c|c|c|}
\hline \multirow[b]{2}{*}{ Valoración WebCT } & \multirow{2}{*}{$\begin{array}{l}\text { Grado empleo } \\
\text { de } \\
\text { autoevaluación }\end{array}$} & \multicolumn{3}{|c|}{ Descriptivos } & \multirow{2}{*}{$\begin{array}{l}\text { Rangos } \\
\text { Promedio (RP) }\end{array}$} \\
\hline & & $\mathrm{N}$ & M & SD & \\
\hline \multirow[t]{3}{*}{ Dimensión contenidos } & Inferior & 16 & 3.76 & .340 & 49.53 \\
\hline & Intermedio & 41 & 3.78 & .445 & 48.95 \\
\hline & Superior & 58 & 4.03 & .470 & 66.73 \\
\hline \multirow{3}{*}{$\begin{array}{l}\text { Dimensión aspectos } \\
\text { formales y técnicos }\end{array}$} & Inferior & 16 & 3.82 & .349 & 47.75 \\
\hline & Intermedio & 41 & 3.89 & .567 & 52.78 \\
\hline & Superior & 58 & 4.08 & .556 & 64.52 \\
\hline \multirow{3}{*}{$\begin{array}{l}\text { Dimensión } \\
\text { autoevaluaci }\end{array}$} & Inferior & 16 & 3.34 & .472 & 41.03 \\
\hline & Intermedio & 41 & 3.48 & .425 & 52.10 \\
\hline & Superior & 58 & 3.70 & .427 & 66.85 \\
\hline \multirow[t]{3}{*}{ Valoración global } & Inferior & 16 & 4.22 & .396 & 51.66 \\
\hline & Intermedio & 41 & 4.17 & .393 & 46.60 \\
\hline & Superior & 58 & 4.41 & .443 & 67.81 \\
\hline
\end{tabular}

Tabla 11. Prueba Kruskal-Wallis para valoración de WebCT según grado de empleo de autoevaluaciones.

\begin{tabular}{lllll}
\hline & \multicolumn{5}{c}{ Kruskal-Wallis Test } \\
Valoración WebCT & $\mathrm{N}$ & Chi-cuadrado & $\mathrm{gl}$ & $\mathrm{Sig}$. \\
\hline Contenidos & 115 & 8.060 & 2 & .018 \\
Aspectos formales y técnicos & 115 & 4.758 & 2 & .093 \\
P. autoevaluación & 115 & 9.554 & 2 & .008 \\
Valoración global & 115 & 10.466 & 2 & .005 \\
\hline
\end{tabular}

La prueba no paramétrica de Kruskal-Wallis indica que no se encontraron diferencias

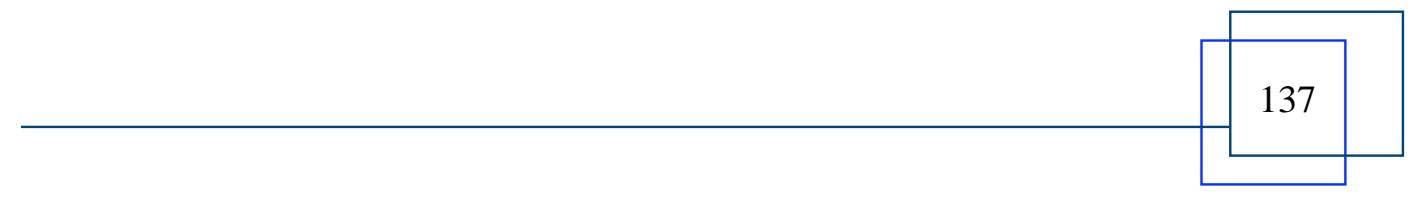




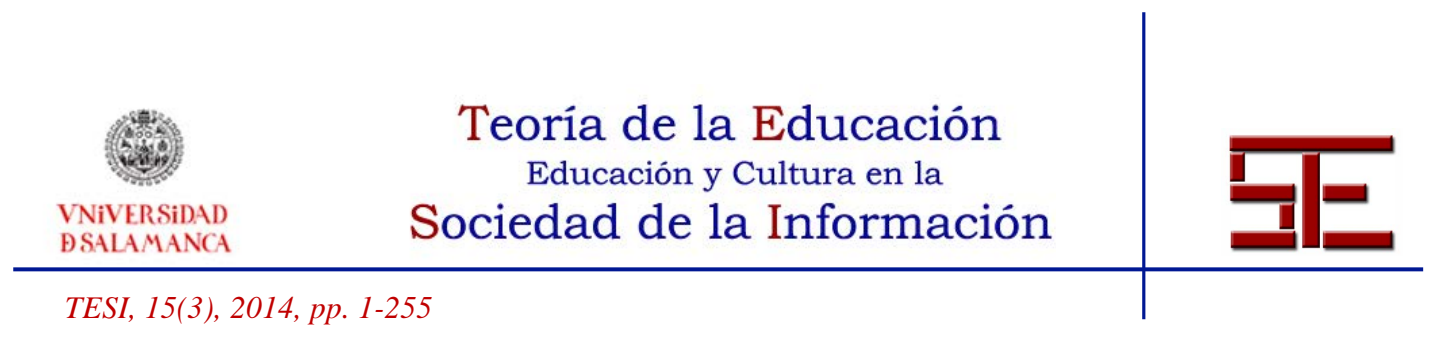

significativas en las valoraciones de los aspectos formales y técnicos de la plataforma, $\mathrm{X}^{2}(2, \mathrm{~N}=115)=4.758, \mathrm{p}=.093$ entre los estudiantes de los tres grados de empleo de las pruebas de autoevaluación; y que se encontraron diferencias significativas en:

- las valoraciones de los contenidos entre los rangos promedio del grado inferior de empleo de las pruebas de autoevaluación $(\mathrm{RP}=49.53)$, del grado intermedio $(\mathrm{RP}=48.95)$ y del grado superior $(\mathrm{RP}=66.73), \mathrm{X}^{2}(2, \mathrm{~N}=115)=8.060, \mathrm{p}=$ .018 ;

- las valoraciones de las pruebas de autoevaluación entre los rangos promedio del grado inferior $(\mathrm{RP}=41.03)$, del grado intermedio $(\mathrm{RP}=52.10)$ y del grado superior $(R P=66.85), X^{2}(2, N=115)=9.554, p=.008$;

- las valoraciones globales de la plataforma entre los rangos promedio del grado inferior $(\mathrm{RP}=51.66)$, del grado intermedio $(\mathrm{RP}=46.60)$ y del grado superior $(\mathrm{RP}=67.81), \mathrm{X}^{2}(2, \mathrm{~N}=115)=10.466, \mathrm{p}=.005$.

Como la prueba realizada no confirma la hipótesis en todas las dimensiones de las valoraciones se acepta la hipótesis nula "No existen diferencias significativas en la percepción y satisfacción del empleo de WebCT por el grado de empleo de las pruebas de autoevaluación" en las valoraciones de los aspectos formales y técnicos de la plataforma. Y se acepta la hipótesis alternativa "Existen diferencias significativas en la percepción y satisfacción del empleo de WebCT por el grado de empleo de las pruebas de autoevaluación" en las valoraciones de los contenidos, pruebas de autoevaluación y en la valoración global.

La tabla 12 recoge las medias y los rangos medios de las valoraciones por dimensiones de WebCT realizada por los estudiantes en función del grado de empleo del foro, y en la tabla 13 el resultado de la prueba de Kruskal-Wallis.

Tabla 12. Descriptivos de la valoración de WebCT por grado de empleo del foro.

\begin{tabular}{|c|c|c|c|c|c|}
\hline \multirow{2}{*}{ Valoración WebCT } & \multirow{2}{*}{$\begin{array}{l}\text { Grado empleo } \\
\text { del foro }\end{array}$} & \multicolumn{3}{|c|}{ Descriptivos } & \multirow{2}{*}{$\begin{array}{l}\text { Rangos } \\
\text { Promedio (RP) }\end{array}$} \\
\hline & & $\mathrm{N}$ & M & SD & \\
\hline \multirow[t]{3}{*}{ Dimensión contenidos } & Inferior & 18 & 3.76 & .420 & 28.97 \\
\hline & Intermedio & 26 & 3.85 & .464 & 32.98 \\
\hline & Superior & 29 & 4.12 & .514 & 45.59 \\
\hline Dimensión & Inferior & 18 & 3.90 & .551 & 32.31 \\
\hline
\end{tabular}

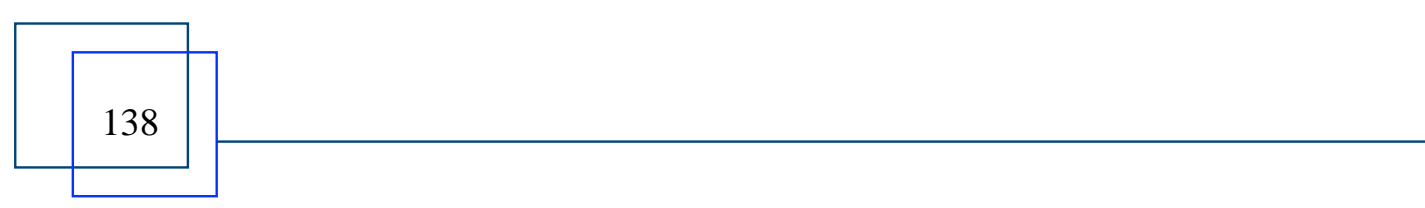




\begin{tabular}{|c|c|c|c|c|c|}
\hline 프 & $\begin{array}{r}\text { Teoría d } \\
\text { Educaci } \\
\text { Sociedad }\end{array}$ & & $\begin{array}{l}\text { Auce } \\
\text { ura er } \\
\text { afor1 }\end{array}$ & ción & 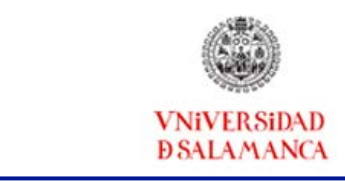 \\
\hline & & & & \multicolumn{2}{|r|}{ TESI, 15(3), 2014, pp. 1-255 } \\
\hline \multirow{2}{*}{ Valoración WebCT } & \multirow{2}{*}{$\begin{array}{l}\text { Grado empleo } \\
\text { del foro }\end{array}$} & \multicolumn{3}{|c|}{ Descriptivos } & Rangos \\
\hline & & $\mathrm{N}$ & $\mathrm{M}$ & SD & Promedio (RP) \\
\hline \multirow[t]{2}{*}{ formales y técnicos } & Intermedio & 26 & 3.85 & .528 & 30.35 \\
\hline & Superior & 29 & 4.27 & .559 & 45.88 \\
\hline \multirow{3}{*}{$\begin{array}{l}\text { Dimensión pruebas } \\
\text { autoevaluación }\end{array}$} & Inferior & 18 & 3.46 & .443 & 31.58 \\
\hline & Intermedio & 26 & 3.54 & .468 & 34.13 \\
\hline & Superior & 29 & 3.71 & .486 & 42.93 \\
\hline \multirow[t]{3}{*}{ Valoración global } & Inferior & 18 & 4.28 & .394 & 32.64 \\
\hline & Intermedio & 26 & 4.28 & .498 & 35.35 \\
\hline & Superior & 29 & 4.44 & .340 & 41.19 \\
\hline
\end{tabular}

Tabla 13. Prueba Kruskal-Wallis para valoración de WebCT según grado de empleo del foro.

\begin{tabular}{lclcl}
\hline & \multicolumn{5}{c}{ Kruskal-Wallis Test } \\
Valoración WebCT & $\mathrm{N}$ & Chi-cuadrado & $\mathrm{gl}$ & $\mathrm{S}$ Sig. \\
\hline Contenidos & 73 & 8.291 & 2 & .016 \\
Aspectos formales y técnicos & 73 & 8.566 & 2 & .014 \\
Pruebas autoevaluación & 73 & 3.928 & 2 & .140 \\
Valoración global & 73 & 2.066 & 2 & .356 \\
\hline
\end{tabular}

La prueba no paramétrica de Kruskal-Wallis indica que no se encontraron diferencias significativas en las valoraciones de las autoevaluaciones, $\mathrm{X}^{2}(2, \mathrm{~N}=73)=3.928, \mathrm{p}=$ .140 y en la valoración global, $\mathrm{X}^{2}(2, \mathrm{~N}=73)=2.066, \mathrm{p}=.356$ entre los estudiantes de los tres grados de empleo del foro; y que se encontraron diferencias significativas en:

- las valoraciones de los contenidos entre los rangos promedio del grado inferior de empleo del foro $(\mathrm{RP}=28.97)$, del grado intermedio $(\mathrm{RP}=32.98)$ y del grado superior $(\mathrm{RP}=45.59), \mathrm{X}^{2}(2, \mathrm{~N}=73)=8.291, \mathrm{p}=.016$; 


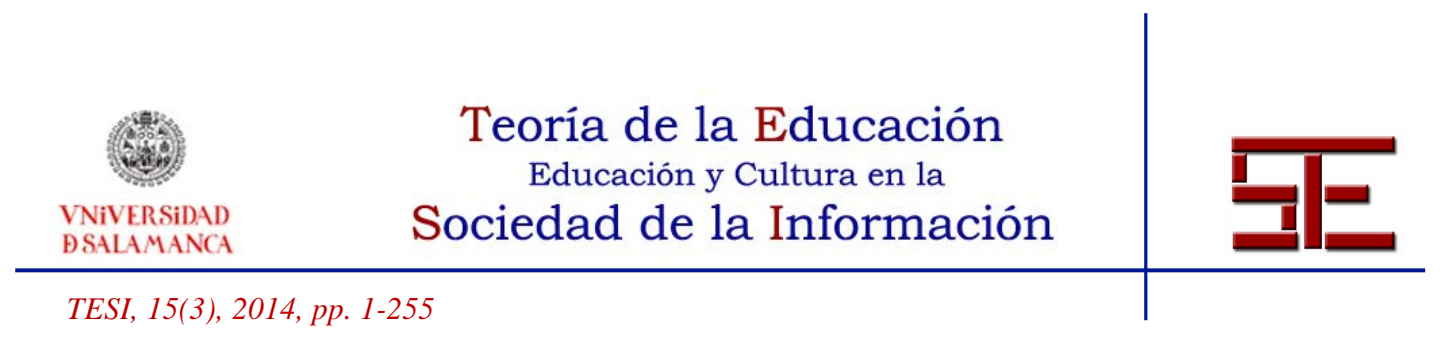

- las valoraciones de los aspectos formales y técnicos entre los rangos promedio del grado inferior $(\mathrm{RP}=32.31)$, del grado intermedio $(\mathrm{RP}=30.35)$ y del grado superior $(\mathrm{RP}=45.88), \mathrm{X}^{2}(2, \mathrm{~N}=73)=8.556, \mathrm{p}=.014$.

Como la prueba realizada no confirma nuestra hipótesis en todas las dimensiones de las valoraciones, se acepta la hipótesis nula "No existen diferencias significativas en la percepción y satisfacción del empleo de WebCT por el grado de empleo del foro" en las valoraciones de las pruebas de autoevaluación y en la valoración global de la plataforma. Y se acepta la hipótesis alternativa "Existen diferencias significativas en la percepción y satisfacción del empleo de WebCT por el grado de empleo del foro" en las valoraciones de los contenidos y de los aspectos formales y técnicos de la plataforma.

\section{CONCLUSIONES}

Los resultados expuestos evidencian que los estudiantes que alcanzan un mayor rendimiento académico son los que presentan una mayor actividad, es decir, aquellos que hacen un uso más frecuente e importante de los medios que a través de WebCT se pone a su disposición.

Se debe destacar que esta metodología ofrece una alta flexibilidad (Bowles, 2004) para adaptarse a las características, adaptándose a las diferencias individuales (Limniou \& Smith, 2010), al ser las plataformas educativas entornos que pueden albergar una gran variedad de medios y recursos que den respuesta a las necesidades de todos los estudiantes.

Es reseñable que la modalidad blended-learning permite un mejor seguimiento del desempeño de los estudiantes (Sitzmann et al., 2006; Vázquez-Martínez, 2011), gracias a las herramientas disponibles en las plataformas educativas, en nuestro caso WebCT lo que se traduce en un aumento del asesoramiento que se les puede ofrecer.

Los estudiantes que mayor uso realizan de la herramienta de comunicación foro en WebCT son quienes mejor valoran los contenidos que disponen en ella de las unidades didácticas así como los aspectos formales y técnicos de la plataforma. De igual manera, los estudiantes que mayor uso realizan de las pruebas de autoevaluación en WebCT son quienes mejor valoran los contenidos y mejor valoración global realizan de la plataforma. Ginns y Ellis (2007) y Webster, Chan, Prosser y Watkins (2009) expusieron que cuando un estudiante tiene una percepción positiva sobre el entorno de aprendizaje tiende a obtener mejores resultados académicos.

Los resultados alcanzados se pueden atribuir a:

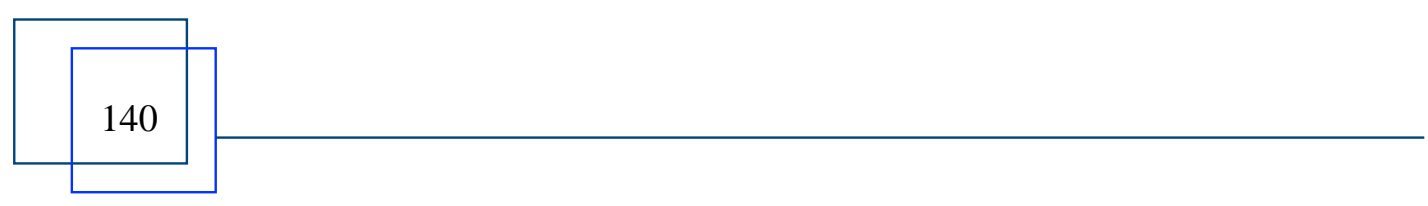




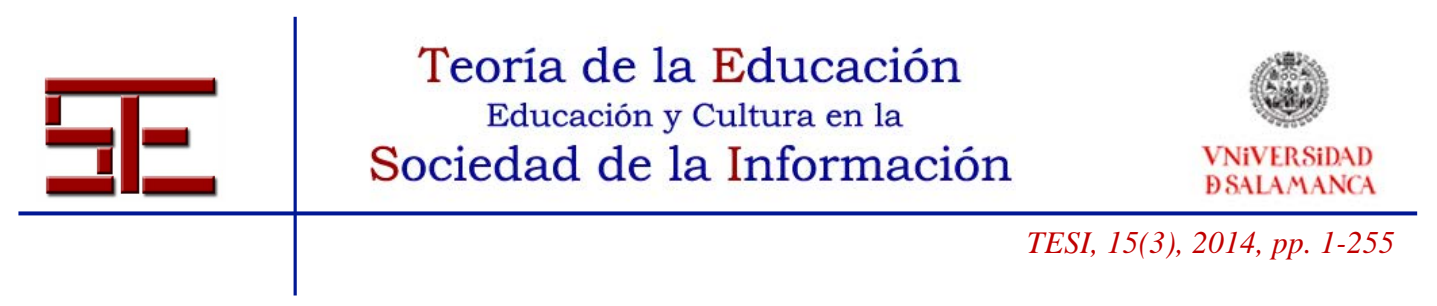

- los recursos de entrenamiento que han tenido los estudiantes a su disposición (Vázquez-Martínez, 2011; Vázquez-Martínez \& Alducin-Ochoa, 2008; Bullen, 2007), y que les ha permitido valorar cuál era el nivel de conocimientos y de progresión en los aprendizajes, y establecer un proceso correcto de autorregulación y monitoreo (Lin \& Wang, 2012; Poon, 2012). Los que obtuvieron los mejores resultados fueron aquellos cuya interacción con el sistema fue mayor (Beer, Clark \& Jones, 2010). El estudiante debía interaccionar con el contenido, con el profesor, con el resto de estudiantes y con los recursos disponibles, y se debe considerar que cuanto mayor sea el grado de interacción mejores serán los conocimientos adquiridos (Sabry \& Balwuin, 2003),

- la interacción con el profesor-tutor, muy superior a la que se dio en la docencia tradicional. Se debe indicar que la asistencia a la tutoría presencial ha sido infrautilizada. Sin embargo, cuando los estudiantes tuvieron a su disposición las herramientas de comunicación, las consultas al profesor-tutor aumentaron de manera importante. Por ello, se podría entender que este ha sido un elemento importante en la mejoría de los resultados (Martínez-Caro \& CampuzanoBolarín, 2011; Lo, 2010). Esta interacción favorece la satisfacción del estudiante (Wu, Tennyson \& Hsia, 2010),

- la interacción entre iguales (Pak \& Verbeke, 2012), desarrollando prácticas colaborativas muy importantes, en tanto a la ayuda prestada entre ellos en los diferentes foros creados para solventar las dudas planteadas por otros compañeros (Moore \& Gilmartin, 2010),

- como resultado de la anterior, para dar respuestas lo más correctas posible a las dudas planteadas por otros compañeros, se observó a lo largo del cuatrimestre cómo los razonamientos y argumentos iban siendo progresivamente de mayor nivel intelectual, con lo que se podía comprobar cómo la comprensión de los contenidos y la capacidad de pensamiento crítico mejoraban (Shannon et al., 2013; Vernadakis, Antoniou, Giannousi, Zetou \& Kiomourtzoglou, 2011; Mosca et al., 2010),

- los estudiantes eran informados al comienzo del curso que todo el trabajo desarrollado fuera del aula sería valorado, con los correspondientes criterios de calificación y evaluación, y por tanto eran conscientes de la importancia de desarrollar un buen hábito de estudio, que les permitiera resolver de manera satisfactoria el proceso de evaluación que sería continua y formativa (VázquezMartínez, 2011; Shirky, 2010), lo que les animaba a esforzarse, pero bajo la premisa que cada uno debía avanzar de acuerdo a su propio ritmo, por lo que la plataforma se adaptaba al progreso de cada uno de ellos, en un proceso de 


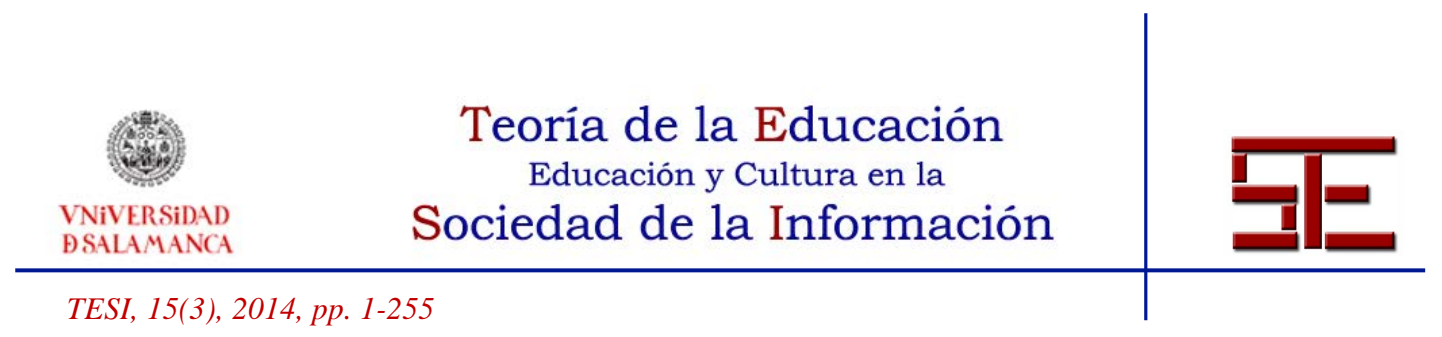

enseñanza-aprendizaje individualizado y flexible (Stricker et al., 2011; Alberts, Murray \& Stephenson, 2010), pero en el que el alumno adquiere una especial responsabilidad sobre su aprendizaje (González et al., 2013; González Rogado et al, 2010).

Como limitaciones debemos reseñar que si bien las opiniones vertidas por los alumnos son importantes desde el punto de vista cuantitativo, quedan huérfanas al no haberse completado en este estudio la vertiente declarativa del mismo, dado que el cuestionario PSEW original es sustancialmente más amplio y recoge más información que la empleada para el desarrollo de esta investigación, y que prevemos actuaría como agente explicativo. Esta limitación es al mismo tiempo una línea de futuro, en la que se considera la investigación cualitativa. La cual será reforzada a través de entrevistas en profundidad con grupos focales que permitan comprender mejor tanto la percepción, ventajas e inconvenientes que los alumnos encuentran a este modelo.

Como líneas de futuro planteamos replicar este estudio en cursos sucesivos, para poder comprobar si se mantienen los hallazgos producidos, lo cual permitiría generalizar los resultados, e incorporar como elementos de interés las estrategias, los estilos y enfoques dominantes que presentan los alumnos. De la misma manera, establecer las rutas de aprendizaje que siguen los alumnos, para identificar cuál/cuáles se muestran como eficaces o ineficaces y relacionarlas con los estilos y enfoques de aprendizaje.

\section{BIBLIOGRAFÍA}

Acosta Lugo, W. (2004): Aproximación a elearning y el Aprendizaje a Distancia. Learning Review, 1. Extraído el 15 marzo, 2005 de <www.learningreview.com.ar>.

Alberts, P. P., Murray, L. A., \& Stephenson, J. E. (2010). Eight educational considerations for hybrid learning. En F. Wang, J. Fong, \& R. Kwan (Eds.), Handbook of research on hybrid learning models: Advanced tools, technologies, and applications (pp. 185-202). Information Sciences Reference: Hershey, PA.

Beer, C., Clark, K. \& Jones, D. (2010). Indicators of Engagement. In Curriculum, Technology \& Transformation for an Unknown Future: Proceedings ASCILITE Sydney 2010. In C. H. Steel, M. J. Keppell, P. Gerbic, \& S. Housego (Ed.), 75-86. Brisbane, Queensland: The University of Queensland. Extraído el 2 enero, $2011 \mathrm{de}<\mathrm{http} / / /$ ascilite. org.au/conferences/sydney10/procs/Beer-full.pdf>. 


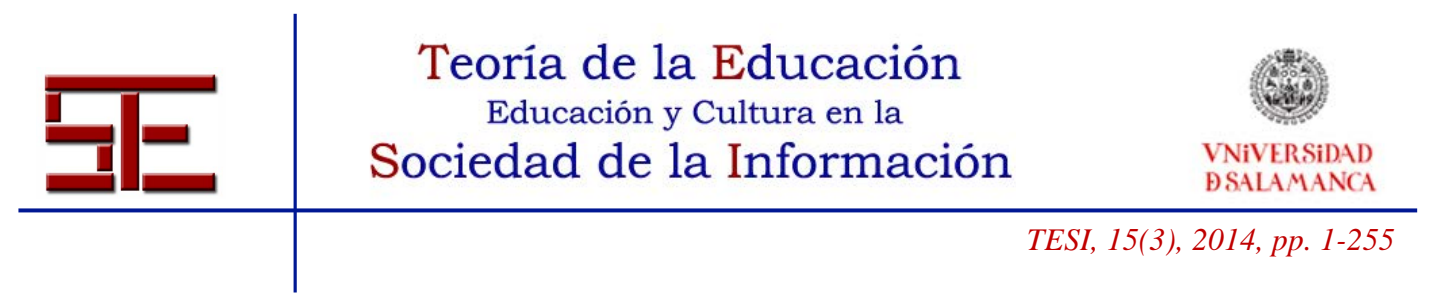

Berlanga, A., Bosom, A. \& Hernández, M. J. (2007). Introducción al e-learning. Recuperado de <http://www.slideshare.net/minicursos/introduccin-al-elearning>.

Bowles, M. S. (2004). Learning to E-Learn Project: Rediscovering the benefits of elearning. Unitas Knowledge Centre. Extraído el 17 diciembre, 2004 de <http://www.marcbowles.com/ifwf/Portals/0/Future\%20and\%20Elearning\%20ICETA2 004.pdf>.

Brindley, J. E., Walti, C. \& Blaschke, L. M. (2009). Creating effective collaborative learning groups in an online environment. International Review of Research in Open and Distance Learning, 10(3), 1-18.

Bullen, P. (September, 2007). Blended Learning Unit. International Conference on Engineering Education - ICEE 2007. Coimbra: Portugal. Extraído el 29 octubre, 2007 de <http://www.ineer.org/events/icee2007/papers/240.pdf>.

Cabero, J. (Dir). (2002). Diseño y evaluación de un material multimedia y telemático para la formación y perfeccionamiento del profesorado universitario para la utilización de las nuevas tecnologías aplicadas a la docencia. Informe final.

Cabero, J. \& Gisbert, M. (2005). La formación en Internet. Sevilla: Eduforma.

Cabero, J. (2007a). La investigación en el ámbito de la Tecnología Educativa. En Cabero Almenara, J. Tecnología Educativa (pp. 249-261). Madrid: McGrawHill.

Cabero, J. (2007b). Comunidades virtuales para el aprendizaje. Su utilización en la enseñanza. Eduweb, 1, 5-22.

Carr-Chellman, A., Dyer, D., \& Breman, J. (2000). Burrowing through the network wires: does distance detract from collaborative authentic learning? Journal of Distance Education, 15(1). Extraído el 24 noviembre, 2000 de <http://cade.athabascau.ca/ vol15.1/carr.html>.

Cheng, K. H. \& Tsai, C. C. (2012). Students' interpersonal perspectives on, conceptions of and approaches to learning in online peer assessment. Australasian Journal of Educational Technology, 28(4), 599-618.

Ching, Y. H. \& Hsu, Y. C. (2011). Design-grounded assessment: A framework and a case study of Web 2.0 practices in higher education. Australasian Journal of Educational Technology, 27(5), 781-797.

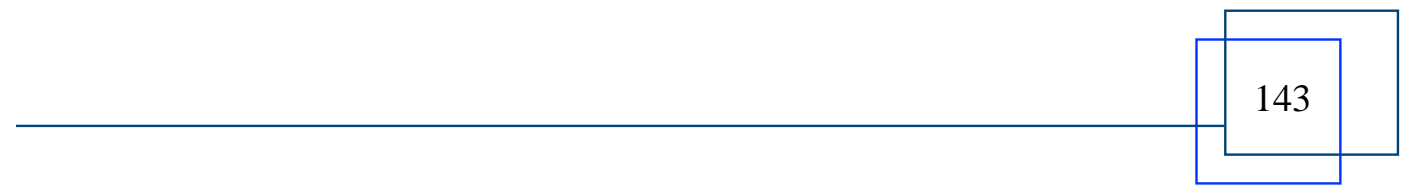




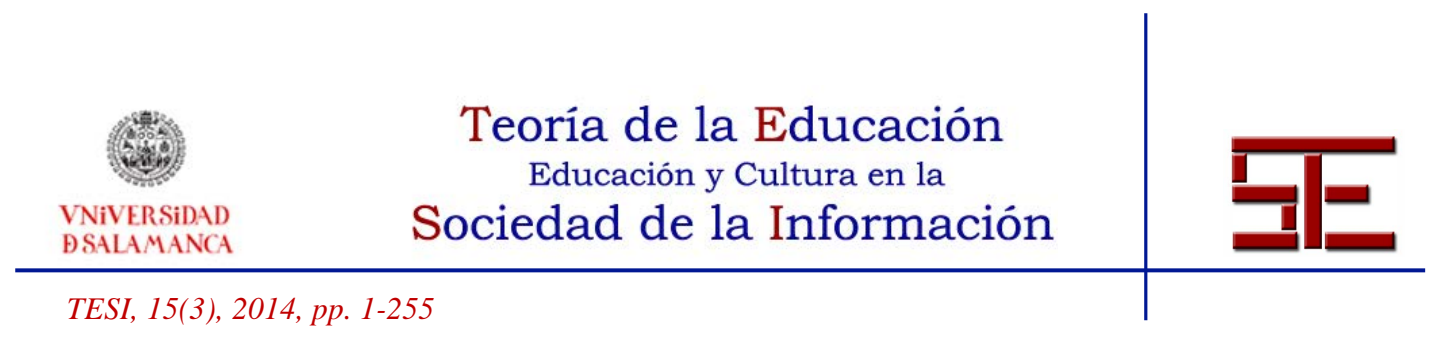

Comisión de las Comunidades Europeas (2000). Comunicación de la Comisión elearning- Concebir la educación del futuro. Bruselas. COM (2000) 318 final. Extraído el 18 septiembre, 2000 de <http://eur-ex.europa.eu/LexUriServ/LexUriServ.do?uri= COM:2000:0318:FIN:ES:PDF>.

Conference of Ministers responsible for Higher Education (2003). Realising the European Higher Education Area. Comunicado de Berlín. Extraído el 19 Octubre, 2003 de

<http://www.ond.vlaanderen.be/hogeronderwijs/bologna/documents/MDC/Berlin_Com munique1.pdf $>$.

Conference of Ministers responsible for Higher Education (2009). The Bologna Process 2020 - The European Higher Education Area in the new decade. Declaración de Leuven and Louvain-la-Neuve. Extraído el 4 mayo, 2009 de <http://www.ond.vlaanderen.be/ hogeronderwijs/bologna/conference/documents/Leuven_Louvain-la-

Neuve_Communiqu\%C3\%A9_April_2009.pdf >.

Cooner, T. S. (2010). Creating opportunities for students in large cohorts to reflect in and on practice: Lessons learnt from a formative evaluation of students' experiences of a technology enhanced blended learning design. British Journal of Educational Technology, 41(2), 271-286.

Davidson, L. K. (2011). A 3-year experience implementing blended TBL: active instructional methods can shift student attitudes to learning. Medical Teacher, 33(9), $750-753$.

De Witt, C. \& Kerres, M. (2003). A didactical framework for the design of blended learning arrangements, Journal of Educational Media, 28(2-3), 101-113.

De Miguel Díaz, M. (Dir.) (2004). Programa de Estudios y análisis destinado a la mejora de la calidad de la enseñanza y de la actividad del profesorado. Dirección General de Universidades. Proyecto EA 2004-0024.

Ellis, R. A., Ginns, P., \& Piggott, L. (2009). E-learning in higher education: Some key aspects and their relationship to approaches to study. Higher Education Research and Development, 28, 303-318.

European University Association (2007). La declaración de Lisboa. Las universidades de Europa más allá de 2010: Diversidad con un propósito común. Extraído el 15 Diciembre, 2007

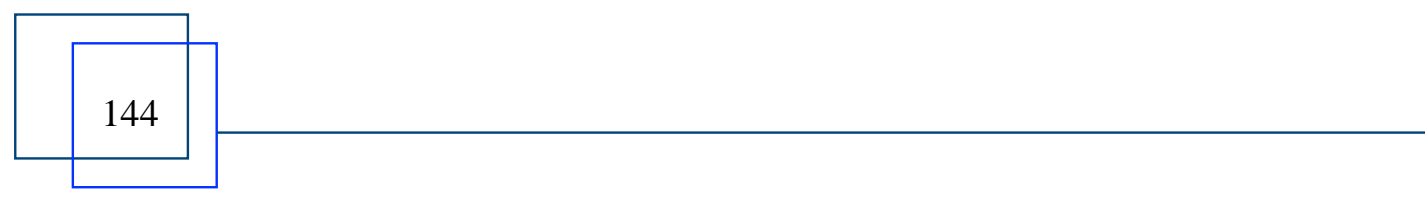




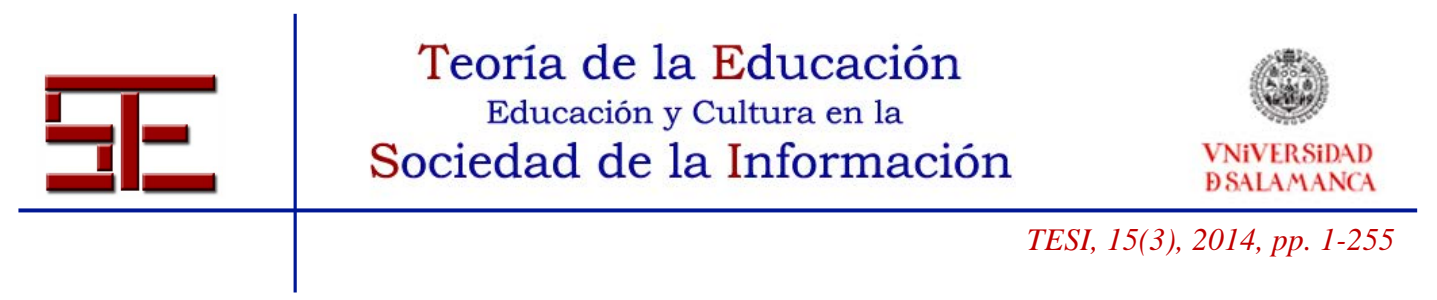

<http://www.eua.be/fileadmin/user_upload/files/Publications/Lisbon_declaration_final_ Spanish_version.pdf $>$.

Francis, R., \& Shannon, S. (2013). Engaging with blended learning to improve students' learning outcomes. European Journal of Engineering Education, 38(4), 1-11.

García Peñalvo, F. J. (2008). Docencia. En J. Laviña Orueta, \& L. Mengual Pavón, Libro Blanco de la Universidad digital 2010 (pp. 29-62). Barcelona: Ariel.

Garrison, D. R., \& Vaughan, N. D. (2008). Blended learning in higher education Framework, principles and guidelines. CA: Jossey-Bass - A Wiley Imprint.

Ginns, P., \& Ellis, R. A. (2009). Evaluating the quality of e-learning at the degree level in the student experience of blended learning. British Journal of Educational Technology, 40(4), 652-663.

George, D., \& Mallery, P. (1995). SPSS/PC+ step by step: a simple guide and referent. Belmont: Wadsworth Publishing Company.

González Rogado, A. B., Rodríguez Conde, M. J., Olmos Migueláñez, S., García Riaza, B., \& García Peñalvo, F. J. (2010). Assessment of a blended-learning methodology in engineering. International Journal of Technology Enhanced Learning, 2(4), 347-357. DOI: 10.1504/IJTEL.2010.035737.

González, A. B., Rodríguez, M. J., Olmos, S., Borham, M., \& García, F. J. (2013). Experimental evaluation of the impact of b-learning methodologies on engineering students in Spain. Computers in Human Behavior, 29(2), 370-377 DOI:10.1016/j.chb.2012.02.003

Harding, A., Kaczynski, D., \& Wood, L. (2005). Evaluation of blended learning: Analysis of qualitative data. Extraído el 17 noviembre, 2005 de <http://ojsprod.library.usyd.edu.au/index.php/IISME/article/viewFile/6436/7085>.

Hölb, M. \& Welzer, T. (2010). Students' feedback and communication habits using Moodle. Electronics and Electrical Engineering, 6(102), 63-66.

Lin, W. S. \& Wang, C. H. (2012). Antecedences to continued intentions of adopting elearning systems in blended learning instruction: A contingency framework based on models of information system success and task-technology fit. Computers in Education, $58,88-99$. 


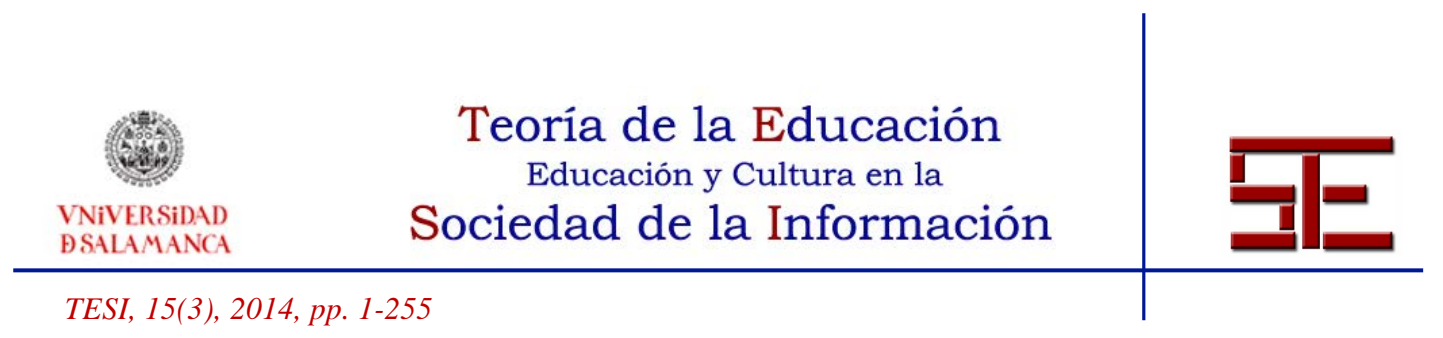

Limniou, M., \& Smith, M. (2010). Teachers' and students' perspectives on teaching and learning through virtual learning environments. European Journal of Engineering Education, 35(6), 645-653.

Lo, C. C. (2010). How student satisfaction factors affect perceived learning. Journal of the Scholarship of Teaching and Learning, 10(1), 47-54.

MacKenzie, N., \& Walsh, A. (2009). Enhancing the curriculum: Shareable multimedia learning objects. Journal of Systems and Information Technology, 11, 71-83.

Martínez-Caro, E. \& Campuzano-Bolarín, F. (2011). Factors affecting students' satisfaction in engineering disciplines: traditional vs. blended approaches. European Journal of Engineering Education, 36(5), 473-483.

Means, B., Toyama, Y., Murphy, R., Bakia, M., \& Jones, K. (2009). Evaluation of Evidence-Based Practices in Online Learning Studies. Washington: U. S. Department of Education, Office of Planning, Evaluation, and Policy Development.

Meurant, R. C. (2010). How computer-based internet-hosted learning management systems such as Moodle can help develop L2 digital literacy. International Journal of Multimedia and Ubiquitous Engineering, 5(2), 1-7.

Ministerio de Educación, Cultura y Deporte (2003). Real Decreto 1125/2003, de 5 de septiembre, por el que se establece el sistema europeo de créditos y el sistema de calificaciones en las titulaciones universitarias de carácter oficial y validez en todo el territorio nacional. $\mathrm{BOE}^{\circ} 224$ de 18 septiembre 2003

Moore, N., \& Gilmartin, M. (2010). Teaching for better learning: A blended learning pilot project with first-year geography undergraduates. Journal of Geography in Higher Education, 34(3), 327-344.

Mosca, J. B., Ball, D. R., Buzza, J. S. \& Paul, D. P. (2010). A Comprehensive Studentbased Analysis of Hybrid Courses: Student Preferences and Design Criteria for Success. Journal of Business and Economics Research, 3(5), 7-21.

Neto, P., Vieira, A., Moreira, B. \& Ribeiro, L. M. (2013). Blended-learning approach in caad: architectural representation and communication focused in teaching architecture and art. International Conference Interactive Collaborative Learning. Extraído el 15 diciembre, $2013 \mathrm{de}<\mathrm{http} / / /$ repositorio-aberto.up.pt/handle/10216/64745>.

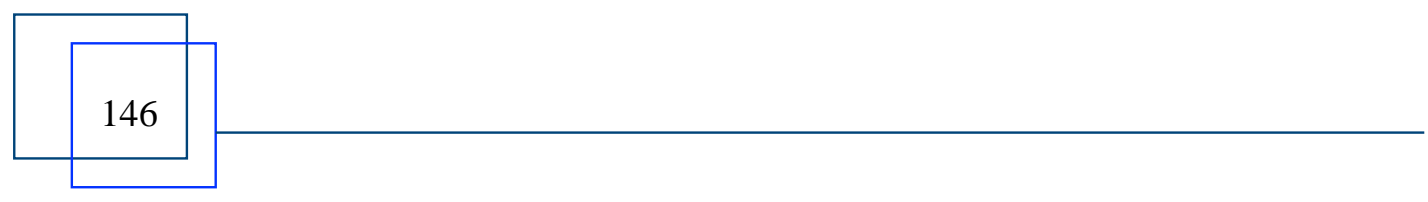




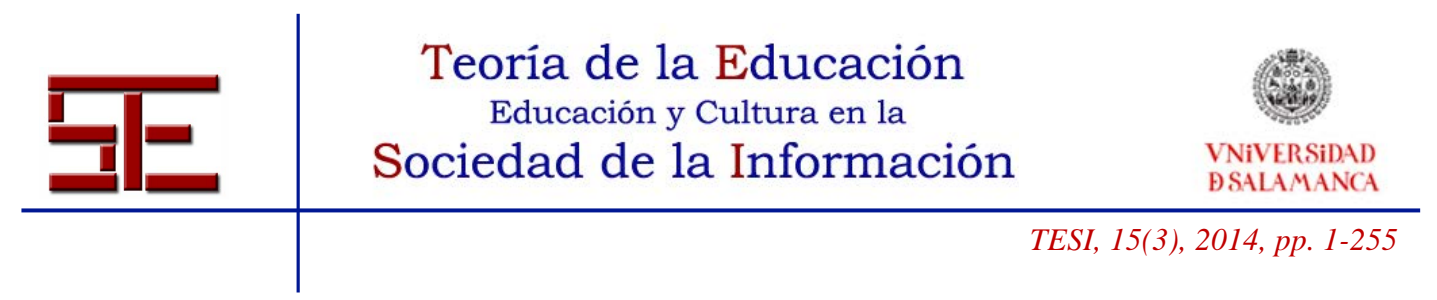

Neumann, D., Neumann, M. \& Hood, M. (2011). Evaluating computerbased simulations, multimedia and animations that help integrate blended learning with lectures in first year statistics. Australasian Journal of Educational Technology, 27(2), 274-289.

Osguthorpe, R. T., \& Graham, C. R. (2003). Blended learning environments. Quarterly Review of Distance Education, 4(3), 227-233.

Pagani, R. \& González, J. (2002). El crédito europeo y el sistema educativo español. Informe técnico. ECTS Counsellors \& Diploma Supplement Promoters.

Pak, B. \& Verbeke, J. (2012). Design studio 2.0: augmenting reflective architectural design learning. ITcon (17), Special Issue eLearning 2.0: Web 2.0-based social learning in built environment, 502-519. Extraído el 14 septiembre, 2012 de <http://www.itcon.org/2012/32>.

Poon, J. (2012). Use of blended learning to enhance the student learning experience and engagement in property education. Property Management, 30(2), 129-156.

Raban, R. \& Litchfield, A. (2007). Supporting peer assessment of individual contributions in groupwork. Australasian Journal of Educational Technology, 23(1), 34-47.

Reisetter, M., Lapointe, L., \& Korcuska, J. (2007). The impact of altered realties: Implications of online delivery for learners' interactions, expectations, and learning skills. International Journal on E- Learning, 6(1), 55-8

Sabry, K. \& Baldwin, L. (2003). Web-based learning interaction and learning styles. British Journal of Educational Technology, 34, 443-454.

Shannon, S. J., Francis , R. L., Leng Chooi, Y. \& Lynn Ng, S. (2012). Approaches to the use of blended learning in teaching tectonics of design to architecture/design and architectural engineering students. Architectural Science Review, 1-10. Extraído el 15 octubre, 2012 de <http://dx.doi.org/10.1080/00038628.2012.744688>.

Shirky, C. (2010). Cognitive Surplus: Creativity and Generosity in a Connected Age. Penguin Press HC. 


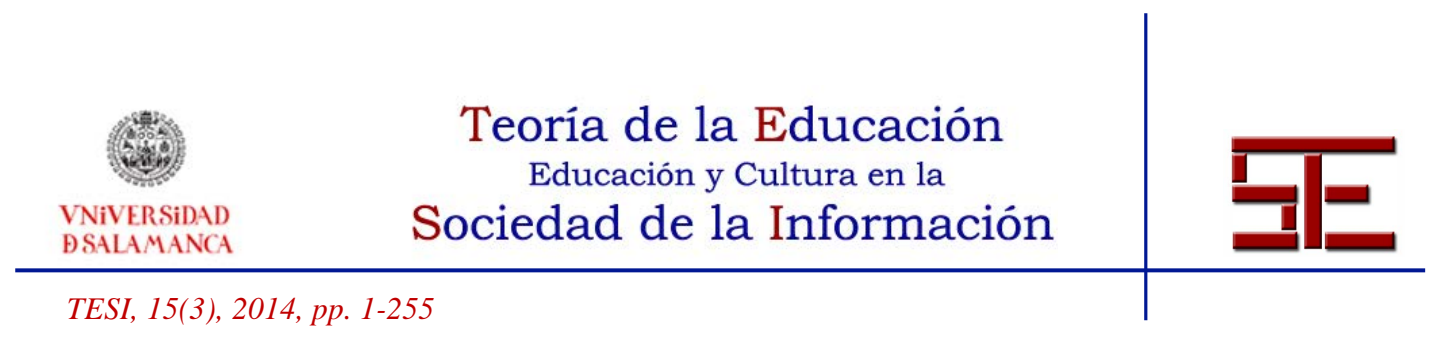

Sitzmann, T. M., Kraiger, K., Stewart, D. W., y Wisher, R. A. (2006). The comparative effectiveness of web-based and classroom instruction: A meta-analysis. Personnel Psychology, 59, 623-664.

Stricker, D., Weible, D. \& Bissmath, B. (2011). Efficient Learning using aVirtual Learning Environment in a University Class. Computers \& Education, 56(2), 495-504.

Vázquez-Martínez, A. I. \& Alducin-Ochoa, J. M. (June, 2008). Mejora del resultado académico a través de entrenamiento en la plataforma educativa WebCT. Paper presented at the $V$ Jornadas de Redes de Investigación en docencia universitaria. Universidad de Alicante: Spain.

Vázquez-Martínez, A. I. (2011). Relación entre los enfoques de aprendizaje y el desempeño de los alumnos en la enseñanza presencial apoyada por plataforma educativa. Estudio de la percepción de los alumnos. Sevilla: Universidad de Sevilla.

Vernadakis, N., Antoniou, P., Giannousi, M., Zetou, E. \& Kioumourtzoglou, E. (2011). Comparing Hybrid Learning with Traditional Approaches on Learning the Microsoft Office Power Point 2003 Program in Tertiary Education. Computers and Education, 56(1), 188-199.

Webster, B. J., Chan, W. S. C., Prosser, M. T. \& Watkins, D. A. (2009). Undergraduates' learning experience and learning process: quantitative evidence from the East. Higher Education, 58(3), 375-386.

Wu, J., Tennyson, R. D., \& Hsia, T. (2010). A study of student satisfaction in a blended e-learning system environment. Computers \& Education, 55, 155-164. 